Алгебра и анализ

Toм $22(2010)$, № 4
St. Petersburg Math. J. Vol. 22 (2011), No. 4, Pages 529-555 S 1061-0022(2011)01157-4

Article electronically published on May 2, 2011

\title{
PARAMETRIZATION OF A TWO-DIMENSIONAL QUASIPERIODIC RAUZY TILING
}

\author{
V. G. ZHURAVLEV
}

\begin{abstract}
With the help of an affine inflation $B$, a two-dimensional quasiperiodic Rauzy tiling $\mathcal{R}^{\infty}$ is constructed, together with a parametrization of its tiles by algebraic integers $\mathbb{Z}[\zeta] \subset[0,1)$, where $\zeta$ is a certain Pisot number (specifically, the real root of the polynomial $x^{3}+x^{2}+x-1$ ). The coronas (clusters) of the tiling $\mathcal{R}^{\infty}$ are classified by disjoint half-intervals in $[0,1)$ the lengths of which are proportional to the frequencies of the corresponding corona types. It is proved that, for each of the three basis tiles, there exists an odd number of corona types of an arbitrary level. The parametrization obtained describes local rules (tile adjacency conditions) for $\mathcal{R}^{\infty}$, and it conjugates the action of the affine rotation $B$ of the plane $\mathbb{R}^{2}$ by an irrational angle with a shift of the coding sequences.
\end{abstract}

\section{INTRODUCTION}

0.1. Finite Rauzy tilings. We denote by $\theta$ the Pisot number equal to the real root of the equation $x^{3}-x^{2}-x-1=0$, and define the shift $S(x)=x+\left(\begin{array}{c}\zeta \\ \zeta^{2}\end{array}\right) \bmod \mathbb{Z}^{2}$ of the torus $\mathbb{T}^{2}=\mathbb{R}^{2} \bmod \mathbb{Z}^{2}$, where $\zeta=\theta^{-1}$. In the paper [1], we constructed a coherent sequence of Rauzy tilings

$$
d^{0} \supset d^{1} \supset \cdots \supset d^{m} \supset \cdots
$$

of level $m$ in which every tile $d^{m+1}$ is obtained by inflation, i.e., by subdividing the tiles of the preceding tiling $d^{m}$. The tilings $d^{m}$ are compatible with $S$, i.e., the action

$$
S: d^{m} \rightarrow S d^{m}
$$

of the shift $S$ on the tiling $d^{m}$ reduces to rearrangement of the basis tiles that partition the region $B^{m} d^{0}$, where $B=\left(\begin{array}{cc}-\zeta & -\zeta \\ 1-\zeta^{2} & -\zeta^{2}\end{array}\right)$. More precisely, the tiling $S d^{m}$ differs from $d^{m}$ only by the position of the three tiles that partition the region $B^{m} d^{0}$. This invariance property of the Rauzy tilings $d^{m}$ is similar to the invariance property of the finite Fibonacci tilings [2], where $S$ is the rotation by the angle $\tau=\frac{1+\sqrt{5}}{2}$ (golden ratio).

In geometric terms, the construction of the Rauzy tilings $d^{m}$ involves partitionable figures or the developments of the torus $\mathcal{T} \simeq \mathbb{T}^{2}$ that admit the tilings

$$
\mathcal{T}=\mathcal{T}_{R}(0)+\mathcal{T}_{G}(v)+\mathcal{T}_{B}(w)
$$

into the tiles $\mathcal{T}_{R}(0)=B \mathcal{T}, \mathcal{T}_{G}(v)=v+\mathcal{T}_{G}(0)$, and $\mathcal{T}_{B}(w)=w+\mathcal{T}_{B}(0)$, where $\mathcal{T}_{G}(0)=$ $B^{2} \mathcal{T}, \mathcal{T}_{B}(0)=B^{3} \mathcal{T}$, and $v$ and $w$ are vectors in $\mathbb{R}^{2}$. The determinant of the matrix $B$ is

2010 Mathematics Subject Classification. Primary 05B45, 52C20, 37B50.

Key words and phrases. Quasiperiodic tilings, local rules, divisible figures, two-dimensional approximations.

Supported by RFBR (grant no. 08-01-00326).

(c)2011 American Mathematical Society 


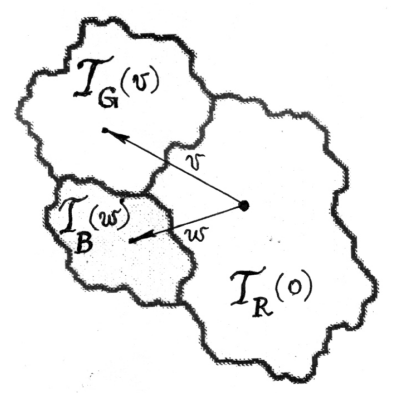

Figure 1. Rauzy tiling $d^{0}$ of zero level

equal to $\zeta$, and since $\zeta+\zeta^{2}+\zeta^{3}=1$, the area of $\mathcal{T}$ is equal to the sum of the areas of the tiles on the right-hand side of equation (0.2). If we take

$$
v=z, \quad w=z+B z, \quad \text { where } \quad z=\left(\begin{array}{c}
\zeta-1 \\
\zeta^{2}
\end{array}\right) \equiv\left(\begin{array}{c}
\zeta \\
\zeta^{2}
\end{array}\right) \bmod \mathbb{Z}^{2}
$$

we obtain a simply connected development of $\mathcal{T}$, and the tiling (0.2) will coincide with the zero level Rauzy tiling $d^{0}$ (Figure 1 ).

0.2. An infinite Rauzy tiling. The Rauzy tiling $\mathcal{R}^{\infty}$ of the plane $\mathbb{R}^{2}$ is constructed by spiral growth beginning with the zero level tiling (0.2):

$$
\mathcal{R}^{0}=\mathcal{T}_{R}(0)+\mathcal{T}_{G}(v)+\mathcal{T}_{B}(w) .
$$

Growth is obtained by blowing up the tiling $\mathcal{R}^{0}\left(\mathcal{R}^{m}=B^{-m} \mathcal{R}^{0}\right)$ in accordance with the following deflation rule (Subsection 6.3):

$$
\begin{aligned}
B^{-1} \mathcal{T}_{R}(0) & =\mathcal{T}_{R}(0)+\mathcal{T}_{G}(v)+\mathcal{T}_{B}(w), \\
B^{-1} \mathcal{T}_{G}(v) & =\mathcal{T}_{R}\left(B^{-1} v\right), \\
B^{-1} \mathcal{T}_{B}(w) & =\mathcal{T}_{G}\left(B^{-1} w\right) .
\end{aligned}
$$

As a result, we obtain the first level tiling

$$
\mathcal{R}^{1}=\left(\mathcal{T}_{R}(0)+\mathcal{T}_{R}\left(B^{-1} v\right)\right)+\left(\mathcal{T}_{G}(v)+\mathcal{T}_{G}\left(B^{-1} w\right)\right)+\mathcal{T}_{B}(w) .
$$

In the general case, the tiling $\mathcal{R}^{m+1}$ is obtained from $\mathcal{R}^{m}$, and the deflation (0.3) has the form

$$
\begin{aligned}
& B^{-1} \mathcal{T}_{R}\left(v_{R}\right)=\mathcal{T}_{R}\left(B^{-1} v_{R}\right)+\mathcal{T}_{G}\left(v+B^{-1} v_{R}\right)+\mathcal{T}_{B}\left(w+B^{-1} v_{R}\right), \\
& B^{-1} \mathcal{T}_{G}\left(v_{G}\right)=\mathcal{T}_{R}\left(B^{-1} v_{G}\right), \\
& B^{-1} \mathcal{T}_{B}\left(v_{B}\right)=\mathcal{T}_{G}\left(B^{-1} v_{B}\right) .
\end{aligned}
$$

The tiling $\mathcal{R}^{m+1}$ is obtained from the tiling

$$
\mathcal{R}^{m}=\sum_{v_{R} \in V_{R}^{m}} \mathcal{T}_{R}\left(v_{R}\right)+\sum_{v_{G} \in V_{G}^{m}} \mathcal{T}_{R}\left(v_{G}\right)+\sum_{v_{B} \in V_{B}^{m}} \mathcal{T}_{R}\left(v_{B}\right)
$$

by the substitutions (0.4),

$$
\mathcal{T}_{R}\left(v_{R}\right) \mapsto B^{-1} \mathcal{T}_{R}\left(v_{R}\right), \quad \mathcal{T}_{G}\left(v_{G}\right) \mapsto B^{-1} \mathcal{T}_{R}\left(v_{G}\right), \quad \mathcal{T}_{R}\left(v_{B}\right) \mapsto B^{-1} \mathcal{T}_{R}\left(v_{B}\right)
$$

Repeating (0.6) three times, we obtain the recurrence relation

$$
\mathcal{R}^{m}=\mathcal{R}^{m-1}+\left(B^{-m} v+\mathcal{R}^{m-2}\right)+\left(B^{-m} w+\mathcal{R}^{m-3}\right)
$$




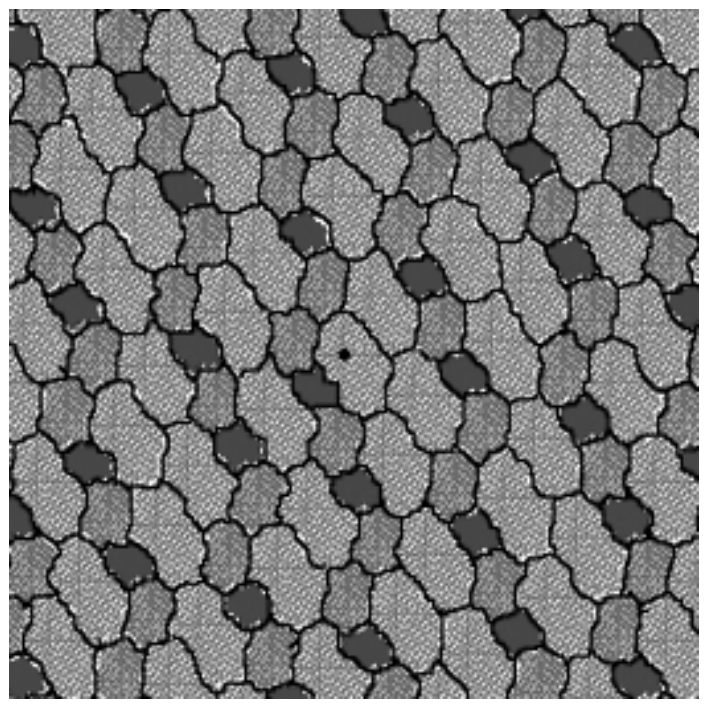

FiguRE 2. A quasiperiodic Rauzy tiling $\mathcal{R}^{\infty}$

with the initial tilings $\mathcal{R}^{-1}=\mathcal{T}_{R}(0), \mathcal{R}^{-2}=\mathcal{T}_{G}(0)$, and $\mathcal{R}^{-3}=\mathcal{T}_{B}(0)$. Since the matrix $B$ determines an affine rotation of the plane, the growth of the tiling $\mathcal{R}^{m}$ is obtained by joining the tilings $\mathcal{R}^{m-2}$ and $\mathcal{R}^{m-3}$ to $\mathcal{R}^{m-1}$ along the spiral (0.7).

In the above construction, we do not use any explicit form of the development of the torus $\mathcal{T}$. Only the tile substitutions (0.6) (and even only the substitutions of their centers) are of importance,

$$
B^{-1}\left\langle v_{R}\right\rangle=B^{-1} v_{R}+V^{0}, \quad B^{-1}\left\langle v_{G}\right\rangle=\left(B^{-1} v_{G}\right)_{R}, \quad B^{-1}\left\langle v_{B}\right\rangle=\left(B^{-1} v_{B}\right)_{G},
$$

where $V^{0}$ consists of $v_{R}=0, v_{G}=v$, and $v_{B}=w$. We identify the set of vectors $V^{m}=V_{R}^{m} \cup V_{G}^{m} \cup V_{B}^{m}$ with the centers of the tiles in the tiling $\mathcal{R}^{m}$ (0.5). From (0.8), we obtain a method of reproducing the centers of tiles, $V^{m+1}=B^{-1}\left\langle V^{m}\right\rangle$, when passing from a level to the next one. This method completely determines the development of the torus

$$
\overline{\mathcal{T}}=\overline{\bigcup_{m=0}^{\infty} B^{m} V^{m}} .
$$

A development of $\mathcal{T}$ contains a neighborhood of zero [18. Therefore, there exists a limiting tiling

$$
\mathcal{R}^{\infty}=\bigcup_{m=0}^{\infty} \mathcal{R}^{m}
$$

of $\mathbb{R}^{2}$ (Figure 2) for the extending sequence of finite tilings $\mathcal{R}^{0} \subset \mathcal{R}^{1} \subset \cdots \subset \mathcal{R}^{m} \subset \cdots$.

Although the tiling $\mathcal{R}^{\infty}$ itself is not periodic, every finite part of it, $\mathcal{R}^{m} \subset \mathcal{R}^{\infty}$, generates a periodic tiling

$$
\mathcal{R}_{\mathbb{Z}^{2}}^{m}=\mathcal{R}^{m}+B^{-m} \mathbb{Z}^{2}
$$

of $\mathbb{R}^{2}$ with the lattice of periods $B^{-m} \mathbb{Z}^{2}$, where $\mathbb{Z}^{2}$ is the set of columns with integral entries. The fundamental region $\mathcal{R}^{m}$ of the tiling $\mathcal{R}_{\mathbb{Z}^{2}}^{m}$ is generated (see 1.17) by the shifts $S^{(m)}=B^{-m} S B^{m} \bmod B^{-m} \mathbb{Z}^{2}$ of the basis tiles $\mathcal{T}_{R}(0), \mathcal{T}_{G}(v)$, and $\mathcal{T}_{B}(w)$. 
The Rauzy tiling $\mathcal{R}^{\infty}$ is quasiperiodic, and, like all such tilings, it admits only local parallel translations. Along with local symmetries, the Rauzy tiling $\mathcal{R}^{\infty}$ has global similarity symmetries, or, in our case, $B$-symmetries. These symmetries manifest themselves in the following two self-similarity properties of the tiling $\mathcal{R}^{\infty}$ :

$$
B \mathcal{R}^{\infty}=\Delta \mathcal{R}^{\infty}, \quad B^{-1} \mathcal{R}^{\infty}=\nabla \mathcal{R}^{\infty} .
$$

Forgetting the coordinates, we can represent the inflation transformation $\mathcal{R}^{\infty} \rightarrow \Delta \mathcal{R}^{\infty}$ as the replacement of the tiles $\mathcal{T}_{R}\left(v_{R}\right)$ by the tilings $v_{R}+B \mathcal{R}^{0}$. The deflation $\mathcal{R}^{\infty} \rightarrow \nabla \mathcal{R}^{\infty}$ is an inverse process in which every connected triple of tiles $\mathcal{T}_{R}\left(v_{R}\right)+\mathcal{T}_{G}\left(v_{G}\right)+\mathcal{T}_{B}\left(v_{B}\right)$ of the form $v+\mathcal{R}^{0}$, where $v$ is a vector, is combined to yield one tile $v_{R}+B^{-1} \mathcal{T}_{R}(0)$.

0.3. Expanding vectors in the basis $\left\{B^{m} z\right\}$. For an arbitrary vector $x \in \mathbb{R}^{2}$, we define the coefficients $\varepsilon_{n}=0$ or 1 , where $n \in \mathbb{Z}$, by the conditions

$$
\varepsilon_{n}=0 \text { if } B^{-n} x \in \mathcal{T}_{R}(*) \text {, and } \varepsilon_{n}=1 \text { otherwise. }
$$

We consider the following sequence infinite on the right:

$$
\varepsilon=\varepsilon(x)=\varepsilon_{-m} \ldots \varepsilon_{-1} \varepsilon_{0} \varepsilon_{1} \ldots,
$$

where $m$ is the least number for which the point $B^{m} x$ belongs to the tiling $\mathcal{R}^{0}$. The sequences $\varepsilon=\varepsilon(x)$ for $x \in \mathbb{R}^{2}$ form the set $E^{\infty}$ of sequences that do not contain three consecutive 1's. In Theorem 7.1, we prove that, for almost all $x \in \mathbb{R}^{2}$ in Lebesgue measure, we have an expansion

$$
x=\varepsilon_{-m} B^{-m} z+\cdots+\varepsilon_{-1} B^{-1} z+\varepsilon_{0} B^{0} z+\varepsilon_{1} B^{1} z+\cdots
$$

of $x$ in terms of the basis $\left\{B^{n} z: n \in \mathbb{Z}\right\}$ with coefficients 0 and 1 . The sequence $r(x)=\varepsilon_{-m} \ldots \varepsilon_{-1} \mid \varepsilon_{0} \varepsilon_{1} \ldots$ is called the code of $x$. By the rule

$$
r\left(B^{ \pm 1} x\right)=B^{ \pm 1} r(x),
$$

such codes conjugate the actions of the affine rotations $B^{ \pm 1}$ on $x$ and the translations $B^{ \pm 1}$ (see (7.12) ) of their codes $r(x)$. The inequality

$$
\left|x-\varepsilon_{-m} B^{-m} z+\cdots+\varepsilon_{n-1} B^{n-1} z\right|<c \zeta^{n / 2},
$$

where the vertical lines stand for the length of a vector and the constant $c>0$ does not depend on $x$, indicates links between the expansions (0.14) and two-dimensional approximations (see [13, p. 285] and [14, 15]). For the relationship with $\beta$-expansions, see [10, 11.

0.4. Parametrization of Rauzy tilings. The tiling $\mathcal{R}^{\infty}$ is obtained by taking the centers $v=v_{R}, v_{G}, v_{B}$ of the tiles $\mathcal{T}_{X}(v) \in \mathcal{R}^{\infty}$ as $x$. Here, only the terms $\varepsilon_{-m}, \ldots, \varepsilon_{-1}$, $\varepsilon_{0}, \varepsilon_{1}$ of the sequence $\varepsilon(v)$, which determine the form $X$ of the tile $\mathcal{T}_{X}(v)$ and its center $v$, i.e., the position of the tile in the plane $\mathbb{R}^{2}$, are of importance. Technically, it is more convenient to represent these beginnings of sequences in the form of numbers

$$
t=t(v)=\varepsilon_{1} \zeta+\varepsilon_{0} \zeta^{2}+\varepsilon_{-1} \zeta^{3}+\cdots+\varepsilon_{-m} \zeta^{m+2},
$$

which form the set

$$
T[\zeta]=\mathbb{Z}[\zeta] \cap[0|\ldots| 1)
$$

(see [7]), where $[0|\ldots| 1)=[0, \zeta) \cup\left[\zeta+\zeta^{2}, 1\right)$. The mapping

$$
\mathcal{T}_{X}(v) \rightleftharpoons t(v)
$$

is a bijection between the set of tiles in $\mathcal{R}^{\infty}$ and the set $T[\zeta]$ (see the algorithm in Subsection 6.2), and thus, we obtain a parametrization

$$
\mathcal{T}_{X}(t(v))=\mathcal{T}_{X}(v)
$$


of the tiling $\mathcal{R}^{\infty}$ by a set of algebraic integers $T[\zeta] \subset[0|\ldots| 1)$. The deflation $\mathcal{R}^{\infty} \rightarrow$ $\nabla \mathcal{R}^{\infty}$ (see (0.11)) implies the contraction $\nabla T[\zeta]=\zeta \cdot T[\zeta]$ of the parameter set (Proposition 6.1), and the inflation $\mathcal{R}^{\infty} \rightarrow \Delta \mathcal{R}^{\infty}$ gives rise to the dilation $\Delta T[\zeta]=\zeta^{-1} \cdot T[\zeta]$. Therefore, in our case, the set $T[\zeta]$ plays the role of a "window" of the well-known model set method [16, 17].

We define the corona $\operatorname{cl}\left(\mathcal{T}_{X}(t), r\right)$ of level $r=0,1,2, \ldots$ (or, simply, the $r$-corona) as the union of all tiles $\mathcal{T}_{X^{\prime}}\left(t^{\prime}\right) \in \mathcal{R}^{\infty}$ that can be connected with $\mathcal{T}_{X}(t)$ by a sequence of at most $r$ tiles tangent to each other. Two coronas of the same level are said to be equivalent,

$$
\operatorname{cl}\left(\mathcal{T}_{X_{1}}\left(t_{1}\right), r\right) \sim \operatorname{cl}\left(\mathcal{T}_{X_{2}}\left(t_{2}\right), r\right),
$$

if they can be superposed (made coincide) by a parallel translation. In particular, for $r=0$, the equivalence (0.18) means that $\mathcal{T}_{X_{1}}\left(t_{1}\right)$ and $\mathcal{T}_{X_{2} 1}\left(t_{2}\right)$ are tiles of the same form, $X_{1}=X_{2}$, and for $r=1$ the tiles have the same first local environments. In $\S 6$, it is proved that the parameters $t_{1}$ and $t_{2}$ belong to one and the same half-interval in the partition

$$
[0|\ldots| 1)=\left[0, \zeta^{2}\right)_{R} \cup\left[\zeta^{2}, \zeta\right)_{G} \cup\left[\zeta+\zeta^{2}, 1\right)_{B}
$$

if and only if $X_{1}=X_{2}$. For the level $r=1$, there are 11 different forms of coronas, which are parametrized by a smaller subdivision of half-intervals in (0.19),

$$
\begin{aligned}
{\left[0, \zeta^{2}\right)_{R}=} & {\left[0, \zeta^{5}\right)+\left[\zeta^{5}, \zeta^{4}\right)+\left[\zeta^{4}, \zeta^{4}+\zeta^{5}\right)+\left[\zeta^{4}+\zeta^{5}, \zeta^{3}\right)+\left[\zeta^{3}, \zeta^{3}+\zeta^{5}\right) } \\
& +\left[\zeta^{3}+\zeta^{5}, \zeta^{3}+\zeta^{4}\right)+\left[\zeta^{3}+\zeta^{4}, \zeta^{2}\right), \\
{\left[\zeta^{2}, \zeta\right)_{G}=} & {\left[\zeta^{2}, \zeta^{2}+\zeta^{4}\right)+\left[\zeta^{2}+\zeta^{4}, \zeta^{2}+\zeta^{3}\right)+\left[\zeta^{2}+\zeta^{3}, \zeta\right),\left[\zeta+\zeta^{2}, 1\right)_{B} } \\
= & {\left[\zeta+\zeta^{2}, 1\right) . }
\end{aligned}
$$

If the tiles $\mathcal{T}_{X_{1}}\left(t_{1}\right)$ and $\mathcal{T}_{X_{2}}\left(t_{2}\right)$ have equivalent clusters (0.18) for all levels $r$, then this is possible only for $t_{1}=t_{2}$ (see Proposition 6.4). An important characteristic of the Rauzy tiling $\mathcal{R}^{\infty}$ is the number of half-intervals $p_{\mathcal{R}^{\infty}}(r)$ in the partitions (0.19) and (0.20) and their generalizations of an arbitrary level $r$ (see Proposition 6.3). The rate of growth of the function $p_{\mathcal{R} \infty}(r)$ as $r \rightarrow \infty$ characterizes the complexity of the tiling $\mathcal{R}^{\infty}$ (see [3, 4] for the details).

0.5. Local rules. The tiling $\mathcal{R}^{\infty}$ can be constructed starting with any tile $\mathcal{T}_{X}(t) \in \mathcal{R}^{\infty}$ and surrounding it layerwise by neighboring tiles according to the following local rules of the algorithm of Subsection 6.2. For every half-interval $\left[\mathrm{cl}_{X}, *\right)$ in (0.20), we have its own set of local parameters $\mathbf{s}_{1}, \ldots, \mathbf{s}_{l}$ and the corresponding local vectors $v\left(\mathbf{s}_{1}\right), \ldots, v\left(\mathbf{s}_{l}\right)$. We assume that the parameter $t$ of the tile $\mathcal{T}_{X}(t)$ belongs to the half-interval $\left[\mathrm{cl}_{X}, *\right)$. Then $\mathcal{T}_{X}(t)$ is surrounded by $l=l\left[\mathrm{cl}_{X}, *\right)$ neighboring tiles

$$
\mathcal{T}_{X_{i}}\left(t+\mathbf{s}_{i}\right)=\mathcal{T}_{X_{i}}\left(v(t)+v\left(\mathbf{s}_{i}\right)\right) \quad(i=1, \ldots, l),
$$

where the form of a tile $X_{i}=R, G$, or $B$ is determined by the inclusion of the parameter $t+\mathbf{s}_{i}$ into one of the half-intervals $\left[0, \zeta^{2}\right)_{R},\left[\zeta^{2}, \zeta\right)_{G}$, or $\left[\zeta+\zeta^{2}, 1\right)_{B}$. If the tile $\mathcal{T}_{X}(t)$ has center $v=v(t)$, then its neighboring tiles have centers $v(t)+v\left(\mathbf{s}_{i}\right)$.

Our goal in the present paper is to obtain a parametrization of the infinite Rauzy tiling $\mathcal{R}^{\infty}$ and, on the basis of this parametrization, to derive the local rules (0.21), which are important not only in the study of approximation of numbers, multidimensional continued fractions, the geometry of quasiperiodic tilings, and color dynamics, but also in numerous problems of theoretical and applied crystallography. For example, using the local rules, one can study the growth and dynamics of the shaping of quasicrystals [5, 6] and their complexity function and forcing [3, 4, as well as diffraction on quasicrystals [7] and their similarity symmetries [8]. 
The method of the present paper can be applied [9] to a wide class of quasiperiodic tilings of a plane the constructions of which are based on cubic irrationalities: generalized Rauzy tilings [9], the tilings generated by the Jacobi-Perron substitutions ([14], [13, p. 253]), the Akiyama tilings [10, etc.

The author wants to thank V. Krasil'shchikov who wrote the computer programs for constructing the tilings for the present paper.

\section{§1. T-NUMBER SYSTEM AND RELATED TILINGS}

1.1. T-number system. The Tribonacci numbers $T_{n}$ are defined by the recurrence relation [1]

$$
T_{n+3}=T_{n+2}+T_{n+1}+T_{n}
$$

for $n \geq 0$ and the initial conditions $T_{0}=1, T_{1}=2$, and $T_{2}=4$. Since the inequality $T_{n+1} \leq 2 T_{n}$ is valid for all $n \geq 0$, every $N$ in $\mathbb{N}=\{0,1,2, \ldots\}$ can be expanded uniquely into the finite sum

$$
N=\sum_{n \geq 0} \varepsilon_{n}(N) T_{n}
$$

where the coefficients $\varepsilon_{n}(N)=0$ or 1 satisfy the condition (cancellation rule)

$$
\varepsilon_{k}(N) \varepsilon_{k+1}(N) \varepsilon_{k+2}(N)=0
$$

for all $k \geq 0$. If $N=0$, then we put $\varepsilon_{n}(N)=0$ for all $n \geq 0$.

The mapping

$$
\mathbb{N} \stackrel{\varepsilon}{\rightleftharpoons} E: N \longmapsto \varepsilon(N)=\left(\varepsilon_{0}(N), \varepsilon_{1}(N), \ldots\right)
$$

is a bijection from the set $\mathbb{N}$ of nonnegative integers onto the set $E$ of sequences $\varepsilon=$ $\left(\varepsilon_{0}, \varepsilon_{1}, \ldots\right)$, where $\varepsilon_{n}=0$ or 1 , satisfying (1.3) and having a finite number of elements $\varepsilon_{n}$ equal to 1 .

1.2. E-tilings of level $m$. We present several methods of constructing tilings on the set $E[1$.

Method 1 involves $E$-coordinates. The tiling

$$
E^{m}=\sum_{\bar{i} \in \bar{I}^{m}} E_{R}^{m}(\bar{i})+\sum_{\bar{j} \in \bar{J}^{m}} E_{G}^{m}(\bar{j})+\sum_{\bar{k} \in \bar{K}^{m}} E_{B}^{m}(\bar{k})
$$

consists of the tiles $E_{R}^{m}(\bar{i}), E_{G}^{m}(\bar{j})$, and $E_{B}^{m}(\bar{k})$ with $E$-coordinates

$$
\bar{i}=(\underbrace{\ldots}_{m} 0), \bar{j}=(\underbrace{\ldots}_{m} 10), \quad \bar{k}=(\underbrace{\ldots}_{m} 11),
$$

which are sequences from $E$ of length $m+1, m+2$, and $m+2$, respectively. The tiles have the form

$$
\begin{aligned}
& E_{R}^{m}(\bar{i})=\left\{\varepsilon \in E:\left.\varepsilon\right|_{m+1}=\bar{i}\right\}, \\
& E_{G}^{m}(\bar{j})=\left\{\varepsilon \in E:\left.\varepsilon\right|_{m+2}=\bar{j}\right\}, \\
& E_{K}^{m}(\bar{k})=\left\{\varepsilon \in E:\left.\varepsilon\right|_{m+2}=\bar{k}\right\},
\end{aligned}
$$

where $\left.\varepsilon\right|_{n}=\left(\varepsilon_{0}, \varepsilon_{1}, \ldots, \varepsilon_{n-1}\right)$ is the beginning of the sequence $\varepsilon$ of length $n$.

Method 2 involves the substitutions (the inflation $E^{m} \longmapsto E^{m+1}$ )

$$
E_{R}^{m}(\bar{i})=E_{R R}^{m}(\bar{i})+E_{R G}^{m}(\bar{i})+E_{R B}^{m}(\bar{i}),
$$


where

$$
\begin{aligned}
& E_{R R}^{m}(\bar{i})=\left\{\varepsilon \in E_{R}^{m}(\bar{i}):\left.\varepsilon\right|_{m+2}=(\bar{i} 0)\right\}, \\
& E_{R G}^{m}(\bar{i})=\left\{\varepsilon \in E_{R}^{m}(\bar{i}):\left.\varepsilon\right|_{m+3}=(\bar{i} 10)\right\}, \\
& E_{R B}^{m}(\bar{i})=\left\{\varepsilon \in E_{R}^{m}(\bar{i}):\left.\varepsilon\right|_{m+3}=(\bar{i} 11)\right\} .
\end{aligned}
$$

In this case, the tiles of $E^{m+1}$ are obtained by the following renaming and subdividing the tiles of $E^{m}$ :

$$
\begin{aligned}
& E_{R}^{m+1}(\bar{i})= \begin{cases}E_{R R}^{m}\left(\bar{i}_{m}\right) & \text { for } \bar{i}=\left(\bar{i}_{m} 0\right) \in \bar{I}^{m+1}, \bar{i}_{m} \in \bar{I}^{m}, \\
E_{G}^{m}\left(\bar{i}^{m}\right) & \text { for } \bar{i} \in \bar{J}^{m} \subset \bar{I}^{m+1} ;\end{cases} \\
& E_{G}^{m+1}(\bar{j})= \begin{cases}E_{R G}^{m}\left(\bar{i}_{m}\right) & \text { for } \bar{j}=\left(\bar{i}_{m} 10\right) \in \bar{J}^{m+1}, \bar{i}_{m} \in \bar{I}^{m}, \\
E_{B}^{m}\left(\bar{j}_{m}\right) & \text { for } \bar{j}=\left(\bar{j}_{m} 0\right) \in \bar{J}^{m+1}, \bar{j}_{m} \in \bar{K}^{m} ;\end{cases} \\
& E_{B}^{m+1}(\bar{k})= \begin{cases}E_{R B}^{m}\left(\bar{i}_{m}\right) & \text { for } \bar{k}=\left(\bar{i}_{m} 11\right) \in \bar{K}^{m+1}, \bar{i}_{m} \in \bar{I}^{m}\end{cases}
\end{aligned}
$$

The zero level tiling $E^{0}$ has the form (1.5): $E^{0}=E_{R}^{0}(0)+E_{G}^{0}(10)+E_{B}^{0}(11)$. On the set $E$, we define a noncommutative operation of juxtaposition $\left(\oplus\right.$-sum) $\varepsilon \oplus \varepsilon^{\prime}=$ $\left(\varepsilon_{0}, \varepsilon_{1}, \ldots, \varepsilon_{m}, \varepsilon_{0}^{\prime}, \varepsilon_{1}^{\prime}, \ldots, \varepsilon_{n}^{\prime}\right)$ if the right-hand sequence satisfies (1.3). Then the set of $E$-coordinates satisfies the recurrence relation

$$
\bar{I}^{m+1}=\bar{I}^{m} \oplus(0) \cup \bar{J}^{m}, \quad \bar{J}^{m+1}=\bar{I}^{m} \oplus(10) \cup \bar{K}^{m} \oplus(0), \quad \bar{K}^{m+1}=\bar{I}^{m} \oplus(11)
$$

for the levels $m \geq 0$, and we put $\bar{I}^{0}=\{(0)\}, \bar{J}^{0}=\{(10)\}, \bar{K}^{0}=\{(11)\}$.

We define more operations on the set $E$, namely, the shift to the right (the multiplicative shift)

$$
E \stackrel{B}{\longrightarrow} E: \varepsilon=\left(\varepsilon_{0}, \varepsilon_{1}, \ldots\right) \longmapsto \vec{\varepsilon}=B(\varepsilon)=\left(0, \varepsilon_{0}, \varepsilon_{1}, \ldots\right) ;
$$

the addition operation $\varepsilon \dot{+} \varepsilon^{\prime}=\varepsilon\left(N+N^{\prime}\right)$ induced by addition of the integers $N, N^{\prime} \in \mathbb{N}$ corresponding to the sequences $\varepsilon$ and $\varepsilon^{\prime}$; and the additive shift

$$
E \stackrel{S}{\longrightarrow} E: \varepsilon \longmapsto S(\varepsilon)=\varepsilon \dot{+}(1) .
$$

Method 3 of constructing $E$-tilings involves the $E$-recurrence relation (cf. (1.1) for the Tribonacci numbers $T_{n}$ )

$$
E^{m}=B E^{m-1}+\left[(1) \dot{+} B^{2} E^{m-2}\right]+\left[(11)+B^{3} E^{m-3}\right]
$$

for $m \geq 0$ and $E^{-1}=E^{-2}=E^{-3}=E$. Using the additive shift $S$, we can represent (1.12) in the form

$$
E^{m+3}=B E^{m+2}+S B^{2} E^{m+1}+S^{3} B^{3} E^{m},
$$

where the mappings $B$ and $S$ (see (1.10) and (1.11) ) do not commute.

1.3. Global coordinates. By the bijection (1.4), we identify the set of $E$-coordinates of the tiles from the tiling $E^{m}$ (see (1.5)) with the sets of $\mathbb{N}$-numbers or coordinates $I^{m}=\varepsilon^{-1}\left(\bar{I}^{m}\right), J^{m}=\varepsilon^{-1}\left(\bar{J}^{m}\right)$, and $K^{m}=\varepsilon^{-1}\left(\bar{K}^{m}\right)$ of the corresponding tiles $E_{R}^{m}(i)=$ $E_{R}^{m}(\bar{i}), E_{G}^{m}(j)=E_{G}^{m}(\bar{j})$, and $E_{B}^{m}(k)=E_{B}^{m}(\bar{k})$, where $\bar{i}=\varepsilon(i)$, etc. Then the sets of $\mathbb{N}$-coordinates satisfy (1.9). Hence,

$$
I^{m}=\left[0, T_{m}\right), \quad J^{m}=\left[T_{m}, T_{m+1}\right), \quad K^{m}=\left[T_{m+1}+T_{m}, T_{m+2}\right),
$$

where the half-interval $\left[T, T^{\prime}\right)$ consists of the integers $T, T+1, \ldots, T^{\prime}-1$. From (1.1), it follows that the sets $I^{m}, J^{m}$, and $K^{m}$ contain, respectively,

$$
T_{m, R}=T_{m}, \quad T_{m, G}=T_{m-1}+T_{m-2}, \quad T_{m, B}=T_{m-1}
$$


numbers, where $m \geq 0, T_{-2}=0$, and $T_{-1}=1$. We introduce the notation $i_{0}=i$, $j_{0}=j-T_{m}$, and $k_{0}=k-T_{m+1}-T_{m}$. Then the tiling (1.5) can be represented in the form

$$
E^{m}=\sum_{i \in I^{m}} E_{R}^{m}(i)+\sum_{j \in J^{m}} E_{G}^{m}(j)+\sum_{k \in K^{m}} E_{B}^{m}(k),
$$

where

$$
\begin{aligned}
& E_{R}^{m}(i)=\bar{i}_{0} \dot{+} E_{R}^{m}(0)=S^{i_{0}} E_{R}^{m}(0), \\
& E_{G}^{m}(j)=\bar{j}_{0}+E_{G}^{m}\left(T_{m}\right)=S^{j_{0}} E_{G}^{m}\left(T_{m}\right), \\
& E_{B}^{m}(k)=\bar{k}_{0} \dot{+} E_{B}^{m}\left(T_{m+1}+T_{m}\right)=S^{k_{0}} E_{B}^{m}\left(T_{m+1}+T_{m}\right) .
\end{aligned}
$$

Relations (1.17) give ground to call the numbers $i, j$, and $k$ the global coordinates of the tiles $E_{R}^{m}(i), E_{G}^{m}(j)$, and $E_{B}^{m}(k)$.

\section{§2. Finite RAuZy tilings}

2.1. Gauge sequence. Let $\mathcal{A}=\{1,2,3\}$ be a finite alphabet, and let $\mathcal{A}^{\text {fin }}$ be the set of finite sequences (words) in the alphabet $\mathcal{A}$. On $\mathcal{A}$, we define the Tribonacci substitution [18 by

$$
\mathcal{A} \stackrel{\Pi}{\longrightarrow} \mathcal{A}^{\mathrm{fin}}: \Pi(1)=12, \Pi(2)=13, \Pi(3)=1
$$

and extend $\Pi$ to the set of sequences $\mathcal{A}^{\text {fin }}$ by putting

$$
\mathcal{A}^{\mathrm{fin}} \stackrel{\Pi}{\longrightarrow} \mathcal{A}^{\mathrm{fin}}: v=v_{1} \ldots v_{m} \mapsto \Pi(v)=\Pi\left(v_{1}\right) \ldots \Pi\left(v_{m}\right)
$$

and $\Pi(\varnothing)=\varnothing$ for the empty word. Similarly, we extend $\Pi$ to the set $\mathcal{A}^{\infty}$ of all finite and infinite sequences: if $v=v_{1} \ldots v_{m} \ldots$ belongs to $\mathcal{A}^{\infty}$, then $\Pi(v)=\Pi\left(v_{1}\right) \ldots \Pi\left(v_{m}\right) \ldots$. The substitution $\Pi$ has a unique fixed sequence

$$
u=12131211213 \cdots=u_{1} u_{2} u_{3} \ldots
$$

We call $u$ the gauge sequence. This is motivated by the fact that the torus winding is kept in a compact region by the sequence $u$.

The initial sequences of $u$ of length $N=0,1,2, \ldots$ are denoted by

$$
U_{N}=u_{1} u_{2} \ldots u_{N}
$$

for $N \geq 1$, and $U_{0}=\varnothing$. For $v=v_{1} \ldots v_{m}$ in $\mathcal{A}^{\text {fin }}$ and $k=1,2,3$ in $\mathcal{A}$, let $r(v)=\left(\begin{array}{c}r_{1}(v) \\ r_{2}(v) \\ r_{3}(v)\end{array}\right)$, where $r_{k}(v)$ is equal to the number of letters $v_{i}=k$ in the sequence $v$.

2.2. The substitution $\Pi$ and Tribonacci numbers. The substitution $\Pi$ acts on the column $r(v)$ by the following rule:

$$
r(\Pi(v))=A \cdot r(v), \quad A=\left(\begin{array}{lll}
1 & 1 & 1 \\
1 & 0 & 0 \\
0 & 1 & 0
\end{array}\right) .
$$

As a consequence of the above formula, we obtain $r\left(\Pi^{n}(v)\right)=A^{n} \cdot r(v)$ for $n=0,1,2, \ldots$, where $\Pi^{n}(v)=\Pi(\ldots(\Pi(v)) \ldots)(n$ times $)$. By the relation

$$
\left(\begin{array}{c}
T_{n+2} \\
T_{n+1} \\
T_{n}
\end{array}\right)=A^{n}\left(\begin{array}{c}
T_{2} \\
T_{1} \\
T_{0}
\end{array}\right)
$$

the substitution matrix $A$ gives rise to the sequence of Tribonacci numbers $T_{n}$, which is defined also by relation (1.1). The characteristic polynomial of the matrix $A$ is equal to $\mathrm{Ch}_{A}(x)=x^{3}-x^{2}-x-1$ and has one real root $\theta \approx 1.8392$ and two complex conjugate 
roots $\alpha$ and $\bar{\alpha}$. Since $\theta>1$ and $|\alpha|^{2}=\frac{1}{\theta}$, we have $|\alpha|=|\bar{\alpha}|<1$; therefore, $\theta$ is a Pisot number. Its inverse $\zeta=\frac{1}{\theta} \approx 0.5436$ satisfies the relation

$$
\zeta+\zeta^{2}+\zeta^{3}=1
$$

2.3. Deviations. For a sequence $v \in \mathcal{A}^{\text {fin }}$, we define the deviations $\Delta_{k}(v)=\zeta^{k} l(v)-$ $r_{k}(v), k=1,2,3$, where $l(v)=r_{1}(v)+r_{2}(v)+r_{3}(v)$ is the length of the sequence $v$. By (2.3), the deviations $\Delta_{k}(v)$ satisfy the linear relation $\Delta_{1}(v)+\Delta_{2}(v)+\Delta_{3}(v)=0$. Therefore, the behavior of $\Delta_{k}(v)$ is described completely by the column vector

$$
\Delta(v)=\left(\begin{array}{c}
\Delta_{1}(v) \\
\Delta_{2}(v)
\end{array}\right)=l(v)\left(\begin{array}{c}
l \zeta \\
\zeta^{2}
\end{array}\right)-\left(\begin{array}{c}
l r_{1}(v) \\
r_{2}(v)
\end{array}\right) .
$$

We note that $\Delta(v)$ is an eigenvector for the substitution $\Pi$,

$$
\Delta(\Pi(v))=B \cdot \Delta(v), \quad \text { where } \quad B=\left(\begin{array}{cc}
-\zeta & -\zeta \\
1-\zeta^{2} & -\zeta^{2}
\end{array}\right)
$$

has the characteristic polynomial

$$
\mathrm{Ch}_{B}(x)=x^{2}+\left(\zeta+\zeta^{2}\right) x+\zeta=(x-\alpha)(x-\bar{\alpha}),
$$

and $\alpha, \bar{\alpha}=\frac{-\left(\zeta+\zeta^{2}\right) \pm i \sqrt{d}}{2}, d=\zeta^{2}+4 \zeta-1>0$.

We introduce a special notation,

$$
\delta(N)=\Delta\left(U_{N}\right)=N\left(\begin{array}{c}
\zeta \\
\zeta^{2}
\end{array}\right)-\left(\begin{array}{c}
r_{1}\left(U_{N}\right) \\
r_{2}\left(U_{N}\right)
\end{array}\right)
$$

for the deviation $\Delta(v)$ in the case of the sequence $v=U_{N}$ (see (2.2) $)$. Then

$$
\delta(N) \equiv N\left(\begin{array}{c}
\zeta \\
\zeta^{2}
\end{array}\right) \bmod \mathbb{Z}^{2}
$$

for $N=0,1,2, \ldots$, so that the mapping $N \mapsto \delta(N)$ yields a winding of the torus $\mathbb{T}^{2}=\mathbb{R}^{2} \bmod \mathbb{Z}^{2}$ obtained by shifting the zero point by the vector $\xi=\left(\begin{array}{c}\zeta \\ \zeta^{2}\end{array}\right) \equiv z \bmod \mathbb{Z}^{2}$, where

$$
z=\delta(1)=\left(\begin{array}{c}
\zeta-1 \\
\zeta^{2}
\end{array}\right)
$$

2.4. Proper sequences. The sequences $v=\Pi^{n}(1)$ for $n=0,1,2, \ldots$, where $\Pi^{0}(1)=1$, are called proper sequences in $\mathcal{A}^{\text {fin }}$. They satisfy the noncommutative recurrence relation (cf. (1.1) and (1.13) )

$$
\Pi^{n+3}(1)=\Pi^{n+2}(1) \Pi^{n+1}(1) \Pi^{n}(1)
$$

for $n \geq 0$ and the initial conditions $\Pi^{0}(1)=1, \Pi^{1}(1)=12$, and $\Pi^{2}(1)=1213$. Since the lengths of these sequences are 1,2, and 4 , respectively, we see that the recurrence relation (2.8) implies that the proper sequences $\Pi^{n}(1)$ have lengths $T_{n}=l\left(\Pi^{n}(1)\right)$ equal to the Tribonacci numbers $T_{n}$ as in (1.1).

Since the relations $\Pi(u)=u$ and $u=1 u^{\prime}$ are valid for the gauge sequence (2.1), we have $u=\Pi^{n}(u)=\Pi^{n}(1) \Pi^{n}\left(u^{\prime}\right)$; therefore, $\Pi^{n}(1)=U_{T_{n}}$ are the initial sequences of length $T_{n}$ for the gauge sequence $u$. From (2.4) it follows that $\Delta\left(\Pi^{n}(1)\right)=B^{n} \Delta(1)$. This fact and relation (2.5) imply the formula

$$
\delta\left(T_{n}\right)=B^{n} z
$$

for $n=0,1,2, \ldots$, where $z$ is the shift (2.7) of the torus.

By induction on $N$, from (2.8) we obtain the following formulas:

$$
r_{k}\left(\Pi^{n}(1)\right)=r_{k}\left(U_{T_{n}}\right)=T_{n-k}
$$

for $k=1,2,3, n \geq 1$, where, by (1.1), we have $T_{-2}=0$ and $T_{-1}=1$. 
By (2.4), the deviation $\delta\left(T_{n}\right)$ is related to the roots of the characteristic equation $\mathrm{Ch}_{B}(x)$ by the formula

$$
\delta\left(T_{n}\right)=\alpha^{n} a+\bar{\alpha}^{n} \bar{a}
$$

for $n=0,1,2, \ldots$, where $a=\left(\begin{array}{c}a_{1} \\ a_{2}\end{array}\right)=\frac{1}{\bar{\alpha}-\alpha}\left(\begin{array}{c}(\zeta-1) \bar{\alpha}+1-2 \zeta \\ \zeta^{2}(\bar{\alpha}-\alpha)+1\end{array}\right)$.

If $N$ has the expansion $N=\sum_{n \geq 0} \varepsilon_{n}(N) T_{n}$, then relations (2.5) and (2.9) imply

$$
\delta(N)=\sum_{n \geq 0} \varepsilon_{n}(N) B^{n} z .
$$

2.5. The Rauzy tilings of level $m$. We consider the subset $D=\delta(E)$ of $\mathbb{R}^{2}$ where, for $\varepsilon=\left(\varepsilon_{0}, \varepsilon_{1}, \ldots\right) \in E$, the mapping $\delta$ is defined by

$$
\delta(\varepsilon)=\sum_{n=0}^{\infty} \varepsilon_{n} B^{n} z .
$$

Here $B$ is the matrix as in (2.4) and $z=\delta(1)$ is the shift vector of the torus (2.7). Since $\zeta$ and $\zeta^{2}$ are irrational, the mapping $\delta: E \rightleftharpoons D$ is bijective.

The tiling (1.5) induces (see [1]) the tiling $D^{m}=\delta\left(E^{m}\right)$ into the tiles $D_{R}^{m}(i), D_{G}^{m}(j)$, and $D_{B}^{m}(k)$, which are the $\delta$-images of the tiles (1.6). We denote by $d_{R}^{m}(i), d_{G}^{m}(j)$, and $d_{B}^{m}(k)$ the sets of interior points of the closures $\overline{D_{R}^{m}(i)}, \overline{D_{G}^{m}(j)}, \overline{D_{B}^{m}(k)}$, respectively. In [18] it was proved that, for the level $m=0$, the set

$$
d^{0}=d_{R}^{0}(0)+d_{G}^{0}(1)+d_{B}^{0}(3)
$$

contains $D^{0}$ and that its components form a tiling of the torus $\mathbb{T}^{2}=\mathbb{R}^{2} \bmod \mathbb{Z}^{2}$ with the following properties.

1. The tiles $d_{*}^{0}(*)$ in (2.14) are simply connected sets.

2. The sets $d_{R}^{0}(0)+\mathbb{Z}^{2}, d_{G}^{0}(1)+\mathbb{Z}^{2}$, and $d_{B}^{0}(3)+\mathbb{Z}^{2}$ are disjoint.

3. The union of their closures coincides with $\mathbb{R}^{2}$.

4. If $v, v^{\prime} \in \mathbb{Z}^{2}, v \neq v^{\prime}$, and $d_{*}^{0}(*)$ is a tile in the tiling (2.14), then the sets $d_{*}^{0}(*)+v$ and $d_{*}^{0}(*)+v^{\prime}$ are disjoint.

\subsection{Torus shift and rearrangements of tiles. Let}

$$
S(x)=x+\xi \bmod \mathbb{Z}^{2},
$$

where $\xi=\left(\begin{array}{c}\zeta \\ \zeta^{2}\end{array}\right)$ is a shift of the torus $\mathbb{T}^{2}$. From the definition (1.11) of the shift $S$ on the set $E$ of sequences, we obtain the commutative diagram

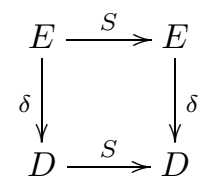

where the lower arrow means the rearrangement of the set $D$ defined by

$$
S(x)=x+z_{R}^{0}, \quad x+z_{G}^{0}, \quad x+z_{B}^{0},
$$

depending on the tile of the tiling $D^{0}=D_{R}^{0}(0)+D_{G}^{0}(1)+D_{B}^{0}(3)$ in which the point $x$ lies. Also, we have

$$
\begin{aligned}
& z_{R}^{0}=\delta(1)-\delta(0)=\xi-\left(\begin{array}{l}
1 \\
0
\end{array}\right), \quad z_{G}^{0}=\delta(2)-\delta(1)=\xi-\left(\begin{array}{l}
0 \\
1
\end{array}\right), \\
& z_{B}^{0}=\delta(4)-\delta(3)=\xi
\end{aligned}
$$

in (2.17). Observe that the restriction of the shift $S$ (see (2.15)) of the torus to the set $D$ coincides with the rearrangement (2.17) $\bmod \mathbb{Z}^{2}$. 


\section{§3. Metrics}

3.1. On the set $\mathbb{N}=\{0,1,2, \ldots\}$, we define an order by putting $\operatorname{ord}(N)=t$ if, in the Tribonacci number system, the number $N \in \mathbb{N}, N \neq 0$, has the expansion $N=$ $\sum_{n \geq t} \varepsilon_{n}(N) T_{n}$ with $\varepsilon_{t}(N) \neq 0$, and $\operatorname{ord}(N)=+\infty$ if $N=0$. This order has the following properties:

$$
\begin{aligned}
\operatorname{ord}\left(N+N^{\prime}\right) & \geq\left\{\operatorname{ord}(N), \operatorname{ord}\left(N^{\prime}\right)\right\}-3, \\
\operatorname{ord}\left(\left|N-N^{\prime}\right|\right) & \geq\left\{\operatorname{ord}(N), \operatorname{ord}\left(N^{\prime}\right)\right\}-2, \\
\operatorname{ord}(\vec{N}) & =\operatorname{ord}(N)+1, \\
\overrightarrow{N+N^{\prime}} & =\vec{N}+\overrightarrow{N^{\prime}} \quad \text { if } \operatorname{ord}(N) \geq 3, \operatorname{ord}\left(N^{\prime}\right) \geq 3, \\
\overrightarrow{\left|N-N^{\prime}\right|} & =\left|\vec{N}-\overrightarrow{N^{\prime}}\right| \quad \text { if } \quad \operatorname{ord}(N) \geq 2, \operatorname{ord}\left(N^{\prime}\right) \geq 2,
\end{aligned}
$$

where

$$
\vec{N}=\sum_{n \geq t} \varepsilon_{n}(N) T_{n+1}
$$

is obtained by a shift of the number $N$ in the system (1.2).

As usual, we define a metric $\varrho$ on the set $\mathbb{N}$ by the formula

$$
\varrho\left(N, N^{\prime}\right)=n\left(\left|N-N^{\prime}\right|\right),
$$

where $n(N)=2^{-\operatorname{ord}(N)}$ is the norm. The metric $\varrho$ has the following properties:

$$
\begin{aligned}
& \varrho\left(N, N^{\prime}\right)>0 \text { for } N \neq N^{\prime}, \quad \varrho\left(N, N^{\prime}\right)=0 \Longleftrightarrow N=N^{\prime}, \\
& \varrho\left(N, N^{\prime}\right)=\varrho\left(N^{\prime}, N\right), \\
& \varrho\left(N, N^{\prime}\right) \leq 8\left(\varrho(N, M)+\varrho\left(M, N^{\prime}\right)\right) .
\end{aligned}
$$

The latter relation is an analog of the triangle inequality.

3.2. By the bijection $\mathbb{N} \stackrel{\varepsilon}{\rightleftharpoons} E$ (see (1.4) $)$, the order ord $(\varepsilon)$, the norm $n(\varepsilon)$, and the metric $\varrho(\varepsilon)$ can be carried over to the set $E$.

3.3. On the torus $\mathbb{T}^{2}$, we define the metric $\varrho(x, y)$ as the distance between the cosets $x+\bmod \mathbb{Z}^{2}, y+\bmod \mathbb{Z}^{2}$ in the Rauzy metric

$$
\|x\|=\left|(\alpha+\zeta) x_{1}+\zeta x_{2}\right| \quad \text { for } \quad x=\left(\begin{array}{l}
x_{1} \\
x_{2}
\end{array}\right) \in \mathbb{R}^{2},
$$

which has the property

$$
\|B x\|=|\alpha| \cdot\|x\|=\zeta^{1 / 2} \cdot\|x\|,
$$

where $B$ is the matrix (2.4).

Proposition 3.1. By continuity, the commutative diagram

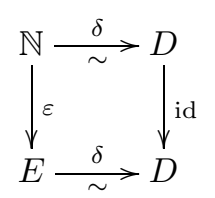


where the mappings $\delta$ are the bijections (2.12) and (2.13) and $\varepsilon$ is the bijection (1.4), can be extended up to the commutative diagram

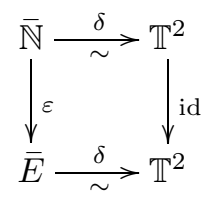

in which all arrows are homeomorphisms.

Proof. The commutativity of (3.7) follows from the relation $\delta(N)=\delta(\varepsilon(N))$, which is a consequence of (2.12) and (2.13). The numbers $1, \zeta$, and $\zeta^{2}$ are linearly independent. Therefore, by (2.6), the set $D=\delta(\mathbb{N})=\delta(E)$ is dense in the torus $\mathbb{T}^{2}$, and, consequently, $\bar{D}=\mathbb{T}^{2}$. To prove that the mappings $\delta$ in (3.8) are homeomorphisms, we need to check that the metrics $\varrho$ on the sets $\mathbb{N}, E$, and $\mathbb{T}^{2}$ are compatible. The fact that the metrics are compatible on $\mathbb{N}$ and $E$ follows from the definition of the metric $\varrho$ on the set $E$. Now, we prove that the metrics $\varrho$ are compatible on the sets $\mathbb{N}$ and $\mathbb{T}^{2}$.

Let $\varrho\left(N, N^{\prime}\right)<\varepsilon$ and, say, $N^{\prime}<N$. Then $N=N^{\prime}+N^{\prime \prime}$, ord $\left(N^{\prime \prime}\right) \geq t$, and $t=t_{\varepsilon} \rightarrow$ $+\infty$ as $\varepsilon \rightarrow 0$. By (2.6), we have $\delta(N) \equiv \delta\left(N^{\prime}\right)+\delta\left(N^{\prime \prime}\right) \bmod \mathbb{Z}^{2}$, whence $\delta(N)-\delta\left(N^{\prime}\right) \equiv$ $\delta\left(N^{\prime \prime}\right) \bmod \mathbb{Z}^{2}$, where

$$
\delta\left(N^{\prime \prime}\right)=\sum_{n \geq t} \varepsilon_{n}\left(N^{\prime \prime}\right) B^{n} z=B^{t}\left(\sum_{n \geq 0} \varepsilon_{n+t}\left(N^{\prime \prime}\right) B^{n} z\right) .
$$

Using (3.9) and the inequality

$$
\|x\|<\frac{1}{2} \quad \text { for all } \quad x \in \bar{D}
$$

(see [18, p. 158]), we obtain $\left\|\delta\left(N^{\prime \prime}\right)\right\|<\frac{1}{2} \zeta^{t / 2}$; consequently, the distance $\varrho\left(\delta(N), \delta\left(N^{\prime}\right)\right)$ $<\frac{1}{2} \zeta^{t / 2}$ tends to zero as $t \rightarrow \infty$, because $0<\zeta<1$.

Conversely, let $\varrho\left(\delta(N), \delta\left(N^{\prime}\right)\right)<\varepsilon^{\prime}$ and $N^{\prime}<N$. Then $N=N^{\prime}+N^{\prime \prime}$, and there exists $l^{\prime} \in \mathbb{Z}^{2}$ such that

$$
\left\|\delta(N)-\delta\left(N^{\prime}\right)-l^{\prime}\right\|<\varepsilon^{\prime} .
$$

By (2.6), we have $\delta(N)-\delta\left(N^{\prime}\right)=N^{\prime \prime} \xi+l^{\prime \prime}$ for some $l^{\prime \prime} \in \mathbb{Z}^{2}$, and then, for $l=l^{\prime}-l^{\prime \prime} \in \mathbb{Z}^{2}$, we have

$$
\left\|N^{\prime \prime} \xi-l\right\|<\varepsilon^{\prime}
$$

by (3.11).

On p. 161 of [18, the following properties of the closure of the development $\bar{D}$ were proved: there exists a constant $c>0$ satisfying the conditions

$$
\begin{aligned}
& \forall x \in \mathbb{R}^{2}:\|x\|<c \Longrightarrow x \in \bar{D} ; \\
& \quad \text { if }\left\|N^{\prime \prime} \xi-l\right\|<c \zeta^{t / 2}, \quad \text { then } \operatorname{ord}\left(N^{\prime \prime}\right) \geq t .
\end{aligned}
$$

From (3.12) and (3.14), we see that if $\varepsilon^{\prime}>0$ is sufficiently small, then $\varrho\left(N, N^{\prime}\right) \leq 2^{-t}<\varepsilon$ for every $\varepsilon>0$ given in advance.

Remark 3.1. The homeomorphism $\overline{\mathbb{N}} \stackrel{\delta}{\rightleftharpoons} \mathbb{T}^{2}$, where $\overline{\mathbb{N}}$ is the closure of $\mathbb{N}$ in the metric (3.3), shows the distinction of this metric from the Euclidean and $p$-adic metrics. In the first case, the closure $\overline{\mathbb{N}}$ coincides with the set $\mathbb{N}$ itself, i.e., is discrete; in the second case, we have $\overline{\mathbb{N}}=\mathbb{Z}_{p}$, where $\mathbb{Z}_{p}$ is the ring of $p$-adic integers, which is compact and totally disconnected. 


\section{$\S 4$. TYPES OF $r$-CORONAS OF RAUZY TILINGS}

4.1. Neighborhood relationship for tiles. Tiles $E_{*}^{m}(i), E_{*}^{m}(j) \in E^{m}$ are said to be neighboring if $\bar{E}_{*}^{m}(i) \cap \bar{E}_{*}^{m}(j) \neq \varnothing$, which is equivalent to

$$
\varrho\left(E_{*}^{m}(i), E_{*}^{m}(j)\right)=\inf _{\substack{\varepsilon \in E_{*}^{m}(i) \\ \varepsilon^{\prime} \in E_{*}^{m}(j)}} \varrho\left(\varepsilon, \varepsilon^{\prime}\right)=0,
$$

where $\varrho$ is the metric (3.3). Similarly, tiles $d_{*}^{m}(i)$ and $d_{*}^{m}(j)$ in the Rauzy tiling $d^{m}$ are neighboring if their closures in the torus metric from Subsection 3.3 satisfy $\bar{d}_{*}^{m}(i) \cap$ $\bar{d}_{*}^{m}(j) \neq \varnothing$.

Proposition 4.1. The bijection $\delta: E \rightleftharpoons D$ (see (2.13) $)$ preserves the neighborhood relationship for tiles.

Proof. The assertion follows from Proposition 3.1.

To consistently define the entire variety of types of local environments for the tiles in the tilings $d^{m}$ of $\mathbb{T}^{2}$, we consider the corresponding periodic tilings $d_{\mathbb{Z}^{2}}^{m}=d^{m}+\mathbb{Z}^{2}$ of $\mathbb{R}^{2}$ obtained by shifting $d^{m}$ by the vectors in $\mathbb{Z}^{2}$. We define the neighborhood relationship for the tiles $d_{*}^{m}(i)+x$ and $d_{*}^{m}(j)+y$, where $x, y \in \mathbb{Z}^{2}$, in the Rauzy metric $\|\cdot\|$ (see (3.5)), which is equivalent to the neighborhood relationship in the Euclidean metric. The difference $s=j-i= \pm 1, \pm 2, \ldots$ will be called the local number of the tile $d_{*}^{m}(j)+y$ with respect to $d_{*}^{m}(i)+x$. By the star st $\left(d_{*}^{m}(i)+x\right)$, we mean the set of all local numbers with respect to the tile $d_{*}^{m}(i)+x$. Since this set does not depend on $x \in \mathbb{Z}^{2}$, we usually drop $x$ in the star notation, writing $\operatorname{st}\left(d_{*}^{m}(i)\right)$. For a fixed tile $d_{*}^{m}(i)$, we define its corona (1-corona) as

$$
\operatorname{cl}\left(d_{*}^{m}(i)\right)=d_{*}^{m}(i) \cup \operatorname{en}\left(d_{*}^{m}(i)\right)
$$

where

$$
\operatorname{en}\left(d_{*}^{m}(i)\right)=\sum_{s \in \operatorname{st}\left(d_{*}^{m}(i)\right)} d_{*}^{m}(i+s)
$$

is the (first) local environment of the tile $d_{*}^{m}(i)$. By the definition in Subsection 2.5, there are three forms of tiles, $R, G$, and $B$. Two tiles $d_{X}^{m}(i)+x$ and $d_{X}^{m}(j)+y$ of the same form have the same local environments if the parallel translation of the plane $\mathbb{R}^{2}$ that superposes these tiles also superposes their clusters. Let the tiles $d_{X}^{m}(i)+x$ and $d_{X}^{m^{\prime}}(j)+y$ have distinct levels $m \neq m^{\prime}$, say, $m>m^{\prime}$. In this case, we also compare their local environments by comparing the local environments of $d_{X}^{m}(i)+x$ and of the tile $B^{m-m^{\prime}}\left(d_{X}^{m^{\prime}}(j)+y\right)$ from the tiling $B^{m-m^{\prime}} d_{\mathbb{Z}^{2}}^{m^{\prime}}$, where $B$ is the matrix in (2.4). The type of the corona $\operatorname{cl}\left(d_{*}^{m}(i)\right)$ is the type of the local environment of its central tile $d_{*}^{m}(i)$.

We begin to classify the types of local environments of the tiling $d^{m}$ starting with the level $m=3$, which is the minimal tiling containing all possible types of local environments for the tilings $d^{m}$ of all levels $m=0,1,2, \ldots$. Another approach to classification of coronas, which involves the kernel $d^{0}$ of the infinite Rauzy tiling (see Figure 1), was used in 3 , 4.

Table 1 contains all possible local environments of the tiling $d^{3}$. This tiling contains 7 tiles of the form $d_{R}^{3}(i), 6$ tiles of the form $d_{G}^{3}(j)$, and 4 tiles of the form $d_{B}^{3}(k)$.

Using $R_{1}^{+}$as an example, we explain the notation employed in Table 1 . The notation $R_{1}^{+}$indicates the type of local environment for the central tile of the form $d_{R}^{3}\left(i_{c}\right)$. The symbol $\left(3^{+}, 3,1\right)$ means that, among its neighbors, the tile $d_{R}^{3}\left(i_{c}\right)$ has three 3 tiles of the form $d_{R}^{3}(*), 3$ tiles of the form $d_{G}^{3}(*)$, and 1 tile of the form $d_{B}^{3}(*)$. The coordination number $7=3+3+1$ is equal to the total number of all tiles that are neighbors for $d_{R}^{3}\left(i_{c}\right)$, and every such tile has its own local number. In their turn, the types of local 


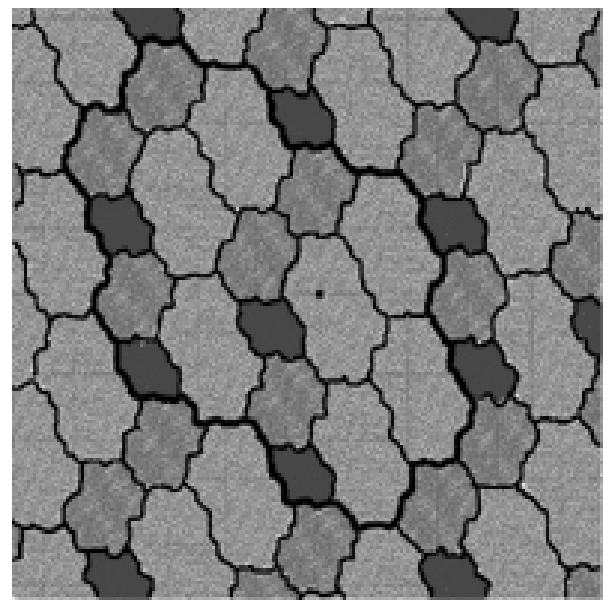

Figure 3 . The periodic Rauzy tiling $d_{\mathbb{Z}^{2}}^{3}$

TABLE 1. Types of coronas of level $m=3$

\begin{tabular}{|c|c|c|c|c|c|c|c|}
\hline $\begin{array}{l}\text { Clust } \\
\text { type }\end{array}$ & $\begin{array}{l}\text { Local environ- } \\
\text { ment symbol }\end{array}$ & $\begin{array}{l}\text { Coord. } \\
\text { number }\end{array}$ & Local numbers $s_{R}$ & \multicolumn{2}{|c|}{$\begin{array}{l}\text { Local } \\
\text { numbers } s_{G}\end{array}$} & \multicolumn{2}{|c|}{$\begin{array}{l}\text { Local } \\
\text { numbers } s_{B}\end{array}$} \\
\hline$R_{1}^{+}$ & $\left(3^{+}, 3,1\right)$ & 7 & $2 \quad 4 \quad 6$ & 9 & & 20 & \\
\hline$R_{1}^{-}$ & $\left(3^{-}, 3,1\right)$ & 7 & $-2-4-6$ & 4 & 6 & 17 & \\
\hline$R_{2}^{+}$ & $\left(\widehat{3}^{+}, 3,1\right)$ & 7 & $\begin{array}{lll}-2 & 2 & 4\end{array}$ & 7 & 9 & 20 & \\
\hline$R_{2}^{-}$ & $\left(\widehat{3}^{-}, 3,1\right)$ & 7 & $-2 \quad 2-4$ & 6 & 7 & 17 & \\
\hline$R_{3}^{+}$ & $\left(2^{+}, 4,1\right)$ & 7 & 24 & 7 & 9 & 20 & \\
\hline$R_{3}^{-}$ & $\left(2^{-}, 4,1\right)$ & 7 & $-2-4$ & 24 & 6 & 17 & \\
\hline$R_{0}^{+}$ & $(2,4,2)$ & 8 & $-2 \quad 2$ & $4 \quad 6$ & 7 & 17 & 20 \\
\hline$G_{1}^{+}$ & $\left(4,1^{+}, 1\right)$ & 6 & $-2-4-6-7$ & 4 & & 13 & \\
\hline$G_{1}^{-}$ & $\left(4,1^{-}, 1\right)$ & 6 & $-6-7-9-11$ & -4 & & 11 & \\
\hline$G_{0}$ & $(4,0,2)$ & 6 & $-4-6-7-9$ & & & 11 & 13 \\
\hline$B$ & $(2,2,0)$ & 4 & $-17-20$ & $-11-$ & -13 & & \\
\hline
\end{tabular}

environments are partitioned into pair environments $\left(R_{1}^{+}, R_{1}^{+}\right),\left(R_{2}^{+}, R_{2}^{+}\right)$, etc. and the unary environments $R_{0}, G_{0}$, and $B_{0}$.

Lemma 4.1. Table 2 contains all types of coronas for tiles of the periodic tilings $d_{\mathbb{Z}^{2}}^{m}$ for the tilings $d^{m}$ of level $m \leq 3$, and every type is determined by the star st of the local numbers $s_{R}, s_{G}$, and $s_{B}$.

Proof. We find the corona types for the tilings $d^{m}, m \leq 3$, by direct inspection of all cases. The procedure can be outlined as follows: 1) first, we look through the tilings $d_{\mathbb{Z}^{2}}^{0}, d_{\mathbb{Z}^{2}}^{1}, d_{\mathbb{Z}^{2}}^{2}$, and $\left.d_{\mathbb{Z}^{2}}^{3} ; 2\right)$ for the tilings $d_{\mathbb{Z}^{2}}^{0}, d_{\mathbb{Z}^{2}}^{1}$, and $d_{\mathbb{Z}^{2}}^{2}$, we use the substitutions (inflation) (1.8), and, for $d_{\mathbb{Z}^{2}}^{3}$, we use the recurrence relation (1.12);3) to check whether tiles from $d_{\mathbb{Z}^{2}}^{m}$ are neighboring, we use diagram (3.8).

Following this plan, we considerably reduce the number of coronas for the tilings $d_{\mathbb{Z}^{2}}^{m}$ through which we must look. Using the tiling $d_{\mathbb{Z}^{2}}^{0}$, where $d^{0}=d_{R}^{0}(0)+d_{G}^{0}(1)+d_{B}^{0}(3)$, as an example, we show how to prove that the closures of the tiles $\bar{d}_{R}^{0}(0), \bar{d}_{G}^{0}(1)$, and $\bar{d}_{B}^{0}(3)$ have a common boundary point. Using diagram (3.8), we replace $d^{0}$ with the $E$-tiling 
TABLE 2. Types of coronas of small levels $m \leq 3$

\begin{tabular}{|c|c|c|c|c|c|c|c|c|c|c|c|c|}
\hline$m$ & Number & $R_{1}^{+}$ & $R_{1}^{-}$ & $R_{2}^{+}$ & $R_{2}^{-}$ & $R_{3}^{+}$ & $R_{3}^{-}$ & $R_{0}$ & $G_{1}^{+}$ & $G_{1}^{-}$ & $G_{0}$ & $B$ \\
\hline 0 & 3 & & & & & $*$ & $*$ & $*$ & $*$ & $*$ & $*$ & $*$ \\
1 & 5 & & & & & $*$ & $*$ & & $*$ & $*$ & & $*$ \\
2 & 8 & $*$ & $*$ & $*$ & $*$ & & & & $*$ & $*$ & $*$ & $*$ \\
3 & 11 & $*$ & $*$ & $*$ & $*$ & $*$ & $*$ & $*$ & $*$ & $*$ & $*$ & $*$ \\
\hline
\end{tabular}

TABLE 3. Ramification of coronas under inflation

\begin{tabular}{|l|l|}
\hline$R_{1}^{+} \mapsto R_{1}^{+}, G_{1}^{+}, B$ & $R_{1}^{-} \mapsto R_{0}, G_{0}, B$ \\
$R_{2}^{+} \mapsto R_{3}^{+}, G_{1}^{+}, B$ & $R_{2}^{-} \mapsto R_{2}^{+}, G_{0}, B$ \\
$R_{3}^{+} \mapsto R_{1}^{+}, G_{1}^{+}, B$ & $R_{3}^{-} \mapsto R_{2}^{+}, G_{0}, B$ \\
$R_{0}^{+} \mapsto R_{3}^{+}, G_{1}^{+}, B$ & \\
\hline$G_{1}^{+} \mapsto R_{2}^{-}$ & $G_{1}^{-} \mapsto R_{1}^{-}$ \\
$G_{0} \mapsto R_{3}^{-}$ & \\
\hline$B \mapsto G_{1}^{-}$ & \\
\hline
\end{tabular}

$E^{0}=E_{R}^{0}(0)+E_{G}^{0}(1)+E_{B}^{0}(3)$. The closures $\bar{E}_{R}^{0}(0), \bar{E}_{G}^{0}(1)$, and $\bar{E}_{B}^{0}(3)$ in the metric $\varrho$ (see Subsection 3.2) contain the the common boundary sequence

$$
\varepsilon=\varepsilon_{0}=\varepsilon_{10}=\varepsilon_{110}
$$

where

$$
\varepsilon_{0}=0001001001 \ldots, \quad \varepsilon_{10}=10001001001 \ldots, \quad \varepsilon_{110}=110001001001 \ldots
$$

are infinite periodic sequences in the closure $\bar{E}$. To prove (4.1), we use two representations of the zero sequence $\overline{0}=000 \ldots$ in the form of a periodic sequence,

$$
\overline{0}_{0}=111011011011 \ldots, \quad \overline{0}_{110}=00111011011011 \ldots .
$$

Observing that $\varepsilon_{0}+\overline{0}_{0}=\varepsilon_{10}$ and $\varepsilon_{110} \dot{+} \overline{0}_{110}=\varepsilon_{10}$, we arrive at (4.1).

4.2. Ramification of coronas under inflation. Method 2 enables us to construct the Rauzy tiling $E^{m+1}$ by means of substitutions (1.8) on tiles of the tiling $E^{m}$. We study the behavior of coronas under the inflation $m \mapsto m+1$.

The behavior of the corona $\operatorname{cl}\left(E_{X}^{m}(*)\right.$ ) (see Subsection 4.1) under the inflation $m \mapsto$ $m+1$ depends on the form of the tile $X=R, G, B$. If $X=R$, then, by the definition (1.8), the tile $E_{R}^{m}(*)=E_{R}^{m+1}(*)+E_{G}^{m+1}(*)+E_{B}^{m+1}(*)$ is subdivided into three new tiles. In the other cases, the tiles change their form, $E_{G}^{m}(*)=E_{R}^{m+1}(*)$ and $E_{B}^{m}(*)=E_{G}^{m+1}(*)$. Thus, the corona $\operatorname{cl}\left(E_{R}^{m}(*)\right)$ splits into three new coronas

$$
\operatorname{cl}\left(E_{R}^{m+1}(*)\right), \quad \operatorname{cl}\left(E_{G}^{m+1}(*)\right), \quad \operatorname{cl}\left(E_{B}^{m+1}(*)\right),
$$

and $E_{G}^{m}(*)$ and $E_{B}^{m}(*)$ are transformed into the coronas

$$
\operatorname{cl}\left(E_{R}^{m+1}(*)\right), \quad \operatorname{cl}\left(E_{G}^{m+1}(*)\right),
$$

respectively. Comparing the local environments listed in Subsection 4.1, we see that the types of coronas (4.2) and (4.3) arising under the inflation $m \mapsto m+1$ depend only on the type of the initial corona $\operatorname{cl}\left(E_{X}^{m}(*)\right)$ and do not depend on its level $m$ admitting the given type of corona.

First, we consider the coronas in Table 1. Looking through all the tiles with the help of diagram (3.8), we find all derivative types of coronas under the inflation $3 \mapsto 4$. Since 
ramification does not depend on $m$, the same types of coronas arise in the general case $m \mapsto m+1$. Table 3 contains all possible types of coronas of Table 1 .

Lemma 4.2. An arbitrary tile $d_{X}^{m}(*)$ in the periodic Rauzy tiling $d_{\mathbb{Z}^{2}}^{m}$ of level $m \geq 3$ has a type of local environment occurring in Table 1, and every such type is represented by a tile $d_{X}^{m}(*)$ in $d_{\mathbb{Z}^{2}}^{m}$.

Proof. We proceed by induction on $m$. For $m=3$, the claim follows from Table 2. By Table 3, no new types arise under the inflation $m \mapsto m+1$, and every corona type in Table 1 arises again under inflation. Thus, Table 1 contains a complete set of all possible corona types that correspond to the tilings $d_{\mathbb{Z}^{2}}^{m}$ of all levels $m=0,1,2, \ldots$, and each tiling $d_{\mathbb{Z}^{2}}^{m}$ of level $m \geq 3$ admits all corona types.

We note that, by Table 2 , the tiling $d_{\mathbb{Z}^{2}}^{3}$ is a periodic tiling of the smallest level containing all corona types (see Figure 3). It can also be proved that, among finite tilings with this property, $d^{5}$ has the smallest possible level.

Theorem 4.1. Consider two tiles $d_{X}^{m}(*)$ and $d_{Y}^{m}(*)$ of the periodic tiling $d_{\mathbb{Z}^{2}}^{m}$ of level $m=0,1,2, \ldots$, where

$$
d^{m}=\sum_{i \in I^{m}} d_{R}^{m}(i)+\sum_{j \in J^{m}} d_{G}^{m}(j)+\sum_{k \in K^{m}} d_{B}^{m}(k)
$$

is the Rauzy tiling (see Subsection 2.5), and the intervals $I^{m}, J^{m}$, and $B^{m}$ are defined by formulas (1.14). These two tiles are neighbors if and only if the following conditions are satisfied:

$$
\begin{array}{llll}
\left|i-i^{\prime}\right| \in I^{m}, & \operatorname{ord}\left(\left|i-i^{\prime}\right|\right) \geq m-2 & \text { for } & d_{R}^{m}(i), d_{R}^{m}\left(i^{\prime}\right) ; \\
|j-i| \in J^{m}, & \operatorname{ord}(|j-i|) \geq m-2 & \text { for } & d_{R}^{m}(i), d_{G}^{m}(j) ; \\
|k-i| \in K^{m}, & \operatorname{ord}(|k-i|) \geq m-1 & \text { for } & d_{R}^{m}(i), d_{B}^{m}(k) ; \\
\left|j-j^{\prime}\right| \in J^{m}, & \operatorname{ord}\left(\left|j-j^{\prime}\right|\right) \geq m-1 & \text { for } & d_{G}^{m}(j), d_{G}^{m}\left(j^{\prime}\right) ; \\
|j-k| \in K^{m}, & \operatorname{ord}(|j-k|) \geq m-1 & \text { for } & d_{G}^{m}(j), d_{B}^{m}(k) ; \\
\left|k-k^{\prime}\right| \in K^{m}, & \operatorname{ord}\left(\left|k-k^{\prime}\right|\right) \geq m-1 & \text { for } & d_{B}^{m}(k), d_{B}^{m}\left(k^{\prime}\right) .
\end{array}
$$

Proof. For the tilings of levels $m=0,1$, and 2, the neighborhood relationship is verified directly. For $m=3$, the claim is justified by Table 1 . Suppose $m \geq 4$. Then, by Lemma 4.2 , every corona $\operatorname{cl}\left(d_{X}^{m}(*)\right)$ has the form $B^{m-3}\left(\operatorname{cl}\left(d_{X}^{3}(*)\right)\right)$ up to a shift. If, say, $s_{3}=j-i$ is a local number of the corona $\operatorname{cl}\left(d_{X}^{3}(*)\right)$, then $s_{m}=\varepsilon^{-1} B^{m-3} \varepsilon\left(s_{3}\right)$ is the corresponding local number of the corona $\operatorname{cl}\left(d_{X}^{m}(*)\right)$, and therefore, its order $\operatorname{ord}\left(s_{m}\right)$ satisfies the inequality indicated in the theorem. If, moreover, the local number $s_{m}$ belongs to the interval conjugate to the inequality indicated, then the tiling $d_{\mathbb{Z}^{2}}^{m}$ admits a corona that is a neighbor of $d_{X}^{m}(*)$.

\subsection{Intervals of types of coronas.}

Theorem 4.2. Let the Rauzy tiling $d^{m}$ have level $m \geq 3$. Then a tile of the form $d_{R}^{m}(i) \in d^{m}$ has a local environment of type $R_{1}^{ \pm}, R_{2}^{ \pm}, R_{3}^{ \pm}, R_{0}$ if and only if its number $i \in I^{m}$ belongs to the corresponding interval

$$
\begin{array}{ll}
I^{m}\left(R_{1}^{+}\right)=\left[0, T_{m-3}\right), & I^{m}\left(R_{1}^{-}\right)=\left[T_{m-1}+T_{m-2}, T_{m}\right), \\
I^{m}\left(R_{3}^{+}\right)=\left[T_{m-3}, T_{m-2}\right), & I^{m}\left(R_{3}^{-}\right)=\left[T_{m-1}+T_{m-3}, T_{m-1}+T_{m-2}\right), \\
I^{m}\left(R_{2}^{+}\right)=\left[T_{m-2}, T_{m-2}+T_{m-3}\right), & I^{m}\left(R_{2}^{-}\right)=\left[T_{m-1}, T_{m-1}+T_{m-3}\right), \\
I^{m}\left(R_{0}\right)=\left[T_{m-2}+T_{m-3}, T_{m-1}\right) . &
\end{array}
$$


The tiles of the form $d_{G}^{m}(j) \in d^{m}$ have local environments of type $G_{1}^{ \pm}, G_{0}$ if their number $j \in J^{m}$ belongs, respectively, to the half-interval

$$
\begin{array}{ll}
J^{m}\left(G_{1}^{+}\right)=\left[T_{m}, T_{m}+T_{m-2}\right), & J^{m}\left(G_{1}^{-}\right)=\left[T_{m}+T_{m-1}, T_{m+1}\right), \\
J^{m}\left(G_{0}\right)=\left[T_{m}+T_{m-2}, T_{m}+T_{m-1}\right) . &
\end{array}
$$

All tiles of the form $d_{B}^{m}(k) \in d^{m}$ have the same type of local environment $B$ for every number $k \in K^{m}$, i.e.,

$$
K^{m}(B)=\left[T_{m+1}+T_{m}, T_{m+2}\right) .
$$

Remark 4.1. For the Rauzy tilings $d^{m}$ of levels $m=0,1,2$, the types of local environment of the tiles $d_{X}^{m}(*)$ can be found with the help of Theorem 4.2 and explicit formulas for the intervals (1.14).

Proof. For the initial levels $m=3,4,5$, formulas (4.5) -4.7) are verified by direct inspection, with detecting the type of local environment for the tile $d_{X}^{m}(*) \in d^{m}$ by its star (see Subsection 4.1)

$$
\operatorname{st}\left(d_{X}^{m}(*)\right)=B^{m-3} \operatorname{st}\left(Y_{*}^{*}\right),
$$

where $\operatorname{st}\left(Y_{*}^{*}\right)$ is the star of the corona of type $R_{1}^{ \pm}, \ldots, B$ consisting of the local numbers $s_{R}, s_{G}$, and $s_{B}$ in Table 1. For example, for the corona type $Y_{*}^{*}=R_{1}^{+}$, its star has the form $\operatorname{st}\left(R_{1}^{+}\right)=\{2,4,6,7,9,11,20\}$.

For the levels $m>5$, we proceed by induction. We consider the tiles $d_{R}^{m}(i), i \in I^{m}$, of a fixed type $\mathcal{R}=R_{1}^{ \pm}, R_{2}^{ \pm}, R_{3}^{ \pm}, R_{0}$. Let

$$
\mathrm{st}^{m}(\mathcal{R})=B^{m-3} \operatorname{st}(\mathcal{R})
$$

be the star of a corona of type $\mathcal{R}$. Then, for a local number $s$ of the form $s_{R}^{m}, s_{G}^{m}, s_{B}^{m} \in$ $\mathrm{st}^{m}(\mathcal{R})$, the relations

$$
i+s_{R}^{m} \in I^{m}, \quad i+s_{G}^{m} \in J^{m}, \quad i+s_{B}^{m} \in K^{m}
$$

provide necessary and sufficient conditions for the tile $d_{R}^{m}(i)$ to have the type $\mathcal{R}$ of local environment. Since the sets $I^{m}, J^{m}$, and $K^{m}$ are half-intervals, conditions (4.8) determine a certain half-interval

$$
I^{m}(\mathcal{R})=\left[I_{\text {min }}^{m}(\mathcal{R}), I_{\text {max }}^{m}(\mathcal{R})\right) \subset I^{m}
$$

for the indices $i$ of the tiles $d_{R}^{m}(i) \in d^{m}$ with local environment of type $\mathcal{R}$. We choose the smallest local numbers $s_{R, \text { min }}^{m} \in \mathrm{st}_{R}^{m}(\mathcal{R}), s_{G, \min }^{m} \in \mathrm{st}_{G}^{m}(\mathcal{R})$, and $s_{B, \text { min }}^{m} \in \operatorname{st}_{B}^{m}(\mathcal{R})$. Let $I_{\mathrm{min}}^{m}, J_{\mathrm{min}}^{m}$, and $K_{\mathrm{min}}^{m}$ be the left endpoints of the half-intervals $I^{m}, J^{m}$, and $K^{m}$, respectively. Then, for every $m$, we have

$$
I_{\min }^{m}(\mathcal{R})=\max \left\{I_{\min }^{m}-s_{R, \min }^{m}, J_{\min }^{m}-s_{G, \min }^{m}, K_{\min }^{m}-s_{K, \min }^{m}\right\} .
$$

Moreover, by Theorem 4.1, we have

$$
\operatorname{ord}\left(s^{m}\right) \geq m-2 \geq 3
$$

for every local number $s^{m} \in \mathrm{st}^{m}(\mathcal{R})$, because $m \geq 5$ by assumption. By (4.10) and formulas (3.1), we obtain the relation

$$
\overrightarrow{I_{\min }^{m}-s_{R, \min }^{m}}=\vec{I}_{\min }^{m}-\vec{s}_{R, \min }^{m},
$$

where $\vec{I}_{\min }^{m}=I_{\min }^{m+1}$ by (1.14) and $\vec{s}_{R, \min }^{m}=s_{R, \text { min }}^{m+1}$ by the definition of the corona type (see Subsection 4.1). Since a similar relation is valid also for the local numbers $s_{G, \min }^{m}$ and $s_{B, \min }^{m}$, equations (4.9) and (4.11) imply

$$
\vec{I}_{\min }^{m}(\mathcal{R})=\max \left\{I_{\min }^{m+1}-s_{R, \min }^{m+1}, J_{\min }^{m+1}-s_{G, \min }^{m+1}, K_{\min }^{m+1}-s_{K, \min }^{m+1}\right\}
$$


where, again by (4.9), the right-hand side is equal to $I_{\min }^{m+1}(\mathcal{R})$. Consequently, $\vec{I}_{\min }^{m}(\mathcal{R})=$ $I_{\min }^{m+1}(\mathcal{R})$, and thus, the left endpoints of the half-intervals $I^{m+1}(\mathcal{R})$ are obtained by shifting the left endpoint of $I^{m}(\mathcal{R})$, which is in agreement with formulas (4.5). For the upper bounds and the coronas of types $G_{1}^{ \pm}, G_{0}$, the proof follows the same lines. Formula (4.7) for coronas of type $B$ stems from Theorem 4.1 and formula (1.14) for the half-intervals $K^{m}$.

\section{§5. Periodic Rauzy tilings}

To classify the coronas, in Subsection 4.1 we introduced the periodic tilings $d_{\mathbb{Z}^{2}}^{m}=$ $d^{m}+\mathbb{Z}^{2}$, where $\mathbb{Z}^{2}$ is the lattice in $\mathbb{R}^{2}$ with the basis $\left(\begin{array}{l}1 \\ 0\end{array}\right),\left(\begin{array}{l}0 \\ 1\end{array}\right)$. The tiling $d_{\mathbb{Z}^{2}}^{m}$ is well defined, because $d^{m}$ is a tiling of the torus $\mathbb{T}^{2}=\mathbb{R}^{2} \bmod \mathbb{Z}^{2}$ (see [1]). In terms of tiles, we can represent the tiling $d_{\mathbb{Z}^{2}}^{m}$ in the form

$$
d_{\mathbb{Z}^{2}}^{m}=\sum_{i \in I^{m}, v \in \mathbb{Z}^{2}} d_{R}^{m}(i, v)+\sum_{j \in J^{m}, v \in \mathbb{Z}^{2}} d_{G}^{m}(j, v)+\sum_{k \in K^{m}, v \in \mathbb{Z}^{2}} d_{B}^{m}(k, v),
$$

where $d_{X}^{m}(i, v)=d_{X}^{m}(i)+v$. For the tile $d_{X}^{m}(i, v)$, its neighbors in the plane $\mathbb{R}^{2}$ are the tiles of its first environment en $\left(d_{X}^{m}(i, v)\right)$ with centers $\delta(i)+v+\delta(s)$ for all local numbers $s$ in the star $\operatorname{st}\left(d_{X}^{m}(i)\right)$, which depends only on the type of the corona $\operatorname{cl}\left(d_{X}^{m}(i)\right)$. By formula (2.6), we have $\delta(i)=i\left(\begin{array}{c}\zeta \\ \zeta^{2}\end{array}\right)-r(i)$, where $r(i)=\left(\begin{array}{c}r_{1}\left(U_{i}\right) \\ r_{2}\left(U_{i}\right)\end{array}\right)$. Therefore,

$$
\delta(i)+\delta(s)=\delta(i+s)-r(i, s)
$$

where $r(i, s)=r(i)+r(s)-r(i+s)$ and, again, the sum $i+s$ belongs to some half-interval $I^{m}, J^{m}$, or $K^{m}$.

Proposition 5.1. We view $d^{m}, m \geq 3$, as a tiling of the development of the torus $\mathcal{T} \simeq \mathbb{T}^{2} \bmod \mathbb{Z}^{2}$ in the plane $\mathbb{R}^{2}$. Then the tile $d_{X}^{m}(i) \in d^{m}$ is a boundary tile if and only if $r(i, s) \neq 0$ for at least one local number $s$ in the star $\operatorname{st}\left(d_{X}^{m}(i)\right)$.

Proof. Since $i+s$ is the index of the tile $d_{X}^{m}(i+s)$ in the tiling $d^{m}$, we see that the tile $d_{X}^{m}(i+s)-r(i, s)$ adjacent to $d_{X}^{m}(i)$ in the plane $\mathbb{R}^{2}$ belongs to the development $\mathcal{T} \subset \mathbb{R}^{2}$ if and only if $r(i, s)=0$, because the vector $r(i, s)$ has integral coordinates.

Proposition 5.2. A periodic Rauzy tiling $d_{\mathbb{Z}^{2}}^{m}$ of level $m \geq 3$ can be constructed starting with an arbitrary tile $d_{X}^{m}(i, v) \in d_{\mathbb{Z}^{2}}^{m}$, and then surrounding it layerwise by adjacent tiles in accordance with the following local rules.

For the tile $d_{X}^{m}(i, v)$, the following tiles are adjacent in the plane $\mathbb{R}^{2}$ :

$$
d_{X}^{m}(i+s, v-r(i, s)) \quad \text { for all local numbers } s \in \operatorname{st}\left(d_{X}^{m}(i)\right) .
$$

The star $\operatorname{st}\left(d_{X}^{m}(i)\right)$ depends on the type $R_{1}^{ \pm}, \ldots, B$ of the local environment of the tile $d_{X}^{m}(i)$ and is defined by the relation

$$
\operatorname{st}\left(d_{X}^{m}(i)\right)=\frac{m-3}{\operatorname{st}\left(d_{X}^{3}(i)\right)}=B^{m-3} \operatorname{st}\left(d_{X}^{3}(i)\right),
$$

where $d_{X}^{3}(i)$ is a tile is $d^{3}$ with the same type of local environment as $d_{X}^{m}(i)$, and the star $\operatorname{st}\left(d_{X}^{3}(i)\right)$ is as in Table 1 . The local environment type for the tile $d_{X}^{m}(i)$ is determined by the half-interval in Theorem 4.2 to which its index $i$ belongs.

Proof. This follows from Lemma 4.2 and identities (5.2). 


\section{§6. Quasiperiodic Rauzy tilings}

6.1. Limit values of local numbers. The passage $\mathcal{R}^{m} \subset \mathcal{R}^{m+1}$ preserves the neighborhood relationship for tiles determined by the local numbers $s^{m}$ and $s^{m+1}$. For the level $m=3$, Table 1 contains all local numbers. In the tiling $d^{m}, m \geq 3$, we choose a tile $d_{R}^{m}(0)$ with local environment of type $R_{1}^{+}$. By Table 1 , its adjacent tiles $d_{G}^{m}\left(T_{m}\right)$ and $d_{B}^{m}\left(T_{m}+T_{m+1}\right)$ have local numbers

$$
s_{G}^{m}=T_{m}=\stackrel{m-3}{\overrightarrow{7}}, \quad s_{B}^{m}=T_{m}+T_{m+1}=\overrightarrow{20} .
$$

In the tiling $d^{m}$, the tile indices change in the half-interval $\left[0, T_{m+2}\right)$. For an infinite tiling $\mathcal{R}^{\infty}$, it is natural to replace the local numbers $s_{X}^{m}$ by their limiting normalized values

$$
\mathbf{s}_{X}=\lim _{m \rightarrow \infty} \frac{s_{X}^{m}}{T_{m+2}} .
$$

From (6.1), we obtain $\mathbf{7}=\zeta^{2}$ and $\mathbf{2 0}=\zeta+\zeta^{2}$. Since $d^{3}$ is a tiling of the smallest level containing clusters of all types, we describe the local numbers and their limit values in the scale $m=3$. To use the limit values $\mathbf{s}$ for tracing the passage to an adjacent tile in the tiling $\mathcal{R}^{\infty}$, we act as in (6.2), normalizing the half-intervals of the corona types from Theorem 4.2 .

The normalized half-intervals $I\left(R_{1}^{+}\right), \ldots, K(B)$ corresponding to the half-intervals $I^{m}\left(R_{1}^{+}\right), \ldots, K^{m}(B)$ are presented in Table 5. The second column contains the lengths of the half-intervals $I\left(R_{1}^{+}\right), \ldots, K(B)$ normalized with respect to the ambient set $[0|\ldots| 1)=$ $[0, \zeta) \cup\left[\zeta+\zeta^{2}, 1\right)$. For example, the normalized length of the half-interval $I\left(R_{1}^{+}\right)$is equal to $\frac{\zeta^{5}}{1-\zeta^{2}} \approx 0.067$. The normalized lengths characterize the relative frequency of the appearance of the corresponding corona type in the Rauzy tiling $\mathcal{R}^{\infty}$.

The tiling $\mathcal{R}^{\infty}$ consists of tiles of the following three types:

$$
\begin{aligned}
& \mathcal{T}_{R}=\mathcal{T}_{R}(0)=B \mathcal{T}, \\
& \mathcal{T}_{G}=\mathcal{T}_{G}(0)=B^{2} \mathcal{T}, \\
& \mathcal{T}_{B}=\mathcal{T}_{B}(0)=B^{3} \mathcal{T},
\end{aligned}
$$

where $\mathcal{T}=\operatorname{int}\left\{\overline{d^{0}}\right\}$ is a development of the torus $\mathbb{T}^{2}$.

TABLE 4. Limiting values of local numbers

\begin{tabular}{|c|c|c|c|c|c|c|c|c|c|}
\hline$s$ & 2 & 4 & 6 & 7 & 9 & 11 & 13 & 17 & 20 \\
\hline $\mathbf{s}$ & $\zeta^{4}$ & $\zeta^{3}$ & $\zeta^{3}+\zeta^{4}$ & $\zeta^{2}$ & $\zeta^{2}+\zeta^{4}$ & $\zeta^{2}+\zeta^{3}$ & $\zeta$ & $\zeta+\zeta^{3}$ & $\zeta+\zeta^{2}$ \\
\hline
\end{tabular}

\subsection{An Algorithm of a layerwise construction of the Rauzy tiling $\mathcal{R}^{\infty}$.}

1. Using Table 5 , around the initial tile $\mathcal{T}_{R}(0)$ with local environment of type $R_{1}^{+}$, we construct the adjacent tiles

$$
\begin{array}{lll}
\mathcal{T}_{R}(\mathbf{2})=\mathcal{T}_{R}(\mathbf{0})+v(2), & \mathcal{T}_{R}(\mathbf{4})=\mathcal{T}_{R}(\mathbf{0})+v(4), & \mathcal{T}_{R}(\mathbf{6})=\mathcal{T}_{R}(\mathbf{0})+v(6), \\
\mathcal{T}_{R}(\mathbf{7})=\mathcal{T}_{R}(\mathbf{0})+v(7), & \mathcal{T}_{R}(\mathbf{9})=\mathcal{T}_{R}(\mathbf{0})+v(9), & \mathcal{T}_{R}(\mathbf{1 1})=\mathcal{T}_{R}(\mathbf{0})+v(11), \\
\mathcal{T}_{R}(\mathbf{2 0})=\mathcal{T}_{R}(\mathbf{0})+v(20), & &
\end{array}
$$

where $v(s)=B^{-3} \delta(s)$ is a local connection vector and $\mathbf{s}$ is the limit value corresponding to the local number $s$. As a result, we obtain the first local environment en(1) of the tile $\mathcal{T}_{R}(0)$. 
TABLE 5. Normalized half-intervals of corona types

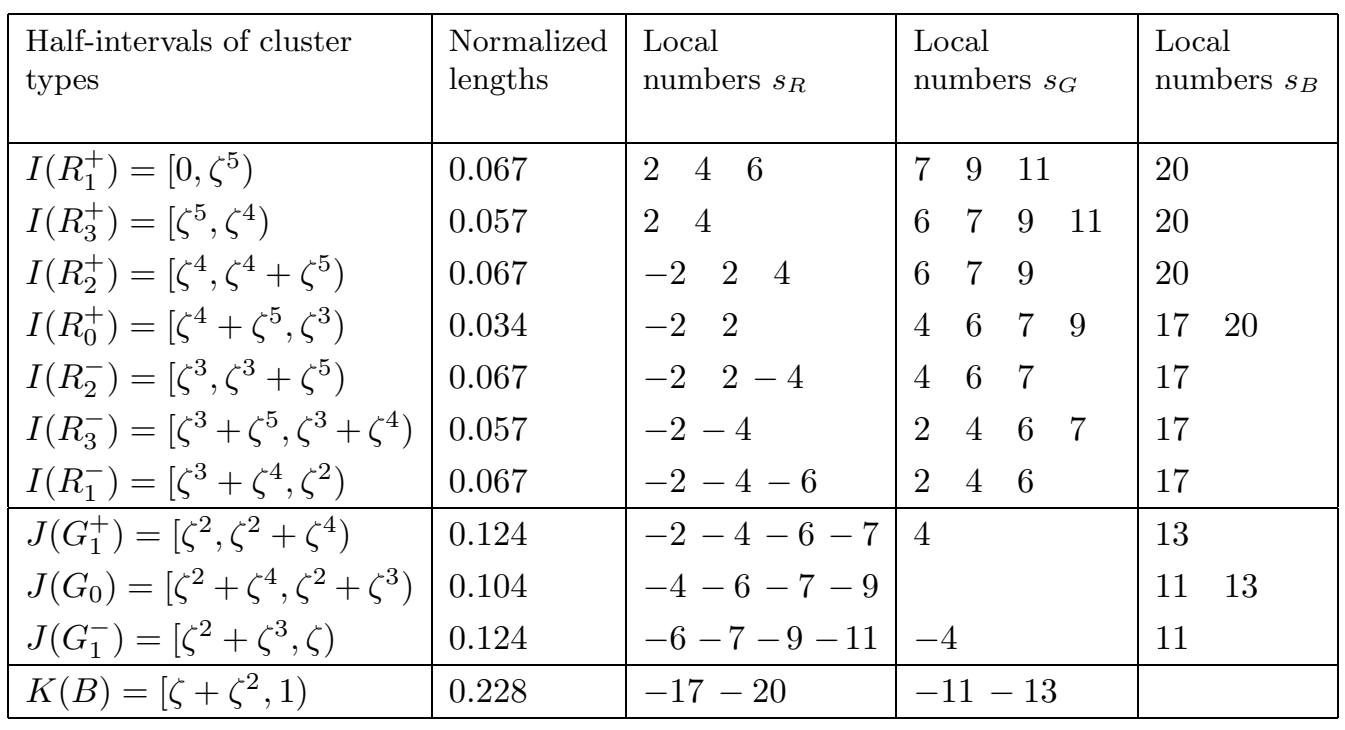

2. To construct the second environment en(2), we append the adjacent tiles to all tiles (6.4). Thus, for example, by Table 4 , the parameter $t=2$ is equal to $t=\zeta^{4}$, and $\zeta^{4} \in I\left(R_{2}^{+}\right)$. Therefore, the tile $\mathcal{T}_{R}(2)$ has the local environment type $R_{2}^{+}$and is surrounded by 7 tiles

$$
\begin{aligned}
& \mathcal{T}_{R}(\mathbf{2}+\mathbf{2})=\mathcal{T}_{R}(\mathbf{0})+v(2)+v(2)=\mathcal{T}_{R}(\mathbf{4}), \\
& \mathcal{T}_{R}(\mathbf{2}-\mathbf{2})=\mathcal{T}_{R}(\mathbf{0})+v(2)-v(2)=\mathcal{T}_{R}(\mathbf{0}), \\
& \ldots \\
& \mathcal{T}_{G}(\mathbf{2}+\mathbf{6})=\mathcal{T}_{G}(\mathbf{0})+v(2)+v(6), \\
& \mathcal{T}_{G}(\mathbf{2}+\mathbf{7})=\mathcal{T}_{G}(\mathbf{0})+v(2)+v(7)=\mathcal{T}_{G}(\mathbf{9}), \\
& \ldots \\
& \mathcal{T}_{B}(\mathbf{2}+\mathbf{2 0})=\mathcal{T}_{B}(\mathbf{0})+v(2)+v(20) .
\end{aligned}
$$

In the sequel, we use the shorthand $\mathcal{T}_{X}(t)=\mathcal{T}_{X}(\mathbf{0})+v\left(s_{1}\right)+\cdots+v\left(s_{r}\right)$, where the parameter $t$ is equal to the sum of the limit values $\mathbf{s}_{\mathbf{1}}+\cdots+\mathbf{s}_{\mathbf{r}} \in[0|\ldots| 1)$ and $s_{1}, \ldots, s_{r}$ is a sequence of compatible local numbers: if for every $k=1, \ldots, r-1$, the sum $\mathbf{s}_{\mathbf{1}}+\cdots+\mathbf{s}_{\mathbf{k}}$ belongs to a half-interval of type $X$, then $s_{k+1}$ must be a local number admitted by the half-interval $X$ (see Table 5). The local environment type for the tile $\mathcal{T}_{X}(t)$ is determined by a half-interval in Table 5 to which the parameter $t$ belongs.

3. Next, we construct the environments en(3), en(4), .., etc.

Justification of the algorithm. For every order $r=0,1,2, \ldots$, there exists a finite tiling $\mathcal{R}^{m} \supset \operatorname{en}\left(\mathcal{T}_{R}(\mathbf{0}), 0\right) \cup \cdots \cup \operatorname{en}\left(\mathcal{T}_{R}(\mathbf{0}), r\right)$, where $\operatorname{en}\left(\mathcal{T}_{R}(\mathbf{0}), r\right)$ is the environment of the initial tile $\mathcal{T}_{R}(\mathbf{0})$ of order $r$ consisting of the tiles of the tiling $\mathcal{R}^{\infty}$ that can be connected with $\mathcal{T}_{R}(\mathbf{0})$ by at least $r$ local vectors $v\left(s_{1}\right), \ldots, v\left(s_{r}\right)$. By the algorithm, the local numbers $s_{1}, \ldots, s_{r}$ are compatible, because the passage from the local numbers $s_{1}^{m}, \ldots, s_{r}^{m}$ to their limit values $\mathbf{s}_{\mathbf{1}}, \ldots, \mathbf{s}_{\mathbf{r}}$ preserves the correspondence between the types of the half-intervals $I^{m}\left(R_{1}^{+}\right), \ldots, K^{m}(B)$ and $I\left(R_{1}^{+}\right), \ldots, K(B)$. It follows that the parameter $t=\mathbf{s}_{\mathbf{1}}+\cdots+\mathbf{s}_{\mathbf{r}}$ belongs to the set $[0|\ldots| 1)$. 
6.3. Deflation and transformation of parameters. We denote by $T[\zeta]$ the set of the values of the parameter $t \in[0|\ldots| 1)$ corresponding to the centers of the tiles $\mathcal{T}_{X}(t)$ of the Rauzy tiling $\mathcal{R}^{\infty}$, which we represent in the form

$$
\mathcal{R}^{\infty}=\sum_{t_{R} \in T_{R}[\zeta]} \mathcal{T}_{R}\left(t_{R}\right)+\sum_{t_{G} \in T_{G}[\zeta]} \mathcal{T}_{G}\left(t_{G}\right)+\sum_{t_{B} \in T_{B}[\zeta]} \mathcal{T}_{B}\left(t_{R}\right) .
$$

Here

$$
T[\zeta]=T_{R}[\zeta]+T_{G}[\zeta]+T_{B}[\zeta]
$$

is partitioned into the subsets $T_{R}[\zeta]=T[\zeta] \cap I, T_{G}[\zeta]=T[\zeta] \cap J$, and $T_{B}[\zeta]=T[\zeta] \cap K$, where (see Table 5) $I=\left[0, \zeta^{2}\right), J=\left[\zeta^{2}, \zeta\right.$ ), and $K=\left[\zeta+\zeta^{2}, 1\right)$ are half-intervals for the tiles of the form $R, G$, and $B$, respectively. From the approximation $\mathcal{R}^{0} \subset \mathcal{R}^{1} \subset \cdots \subset$ $\mathcal{R}^{m} \subset \cdots$ of the tiling $\mathcal{R}^{\infty}$ by finite tilings $\mathcal{R}^{m}$ and the definition of the normalized limit values $\mathbf{s}$, it follows that there exists a bijection between the set of parameters $T[\zeta]$ and the tiles of the tiling $\mathcal{R}^{\infty}$.

Proposition 6.1. Under the deflation $B^{-1}: \mathbb{R}^{2} \longrightarrow \mathbb{R}^{2}$, the parameters of the tiles of $\mathcal{R}^{\infty}$ are transformed by the following ramification rule:

$$
\begin{aligned}
& B^{-1}: t_{R} \mapsto\left\{\begin{array}{l}
t_{R}^{\prime}=\zeta t_{R}, \\
t_{G}^{\prime}=\zeta t_{R}+\zeta^{2}, \\
t_{B}^{\prime}=\zeta t_{R}+\zeta+\zeta^{2},
\end{array}\right. \\
& B^{-1}: t_{G} \mapsto t_{R}^{\prime}=\zeta t_{G}, \\
& B^{-1}: t_{B} \mapsto t_{G}^{\prime}=\zeta t_{B},
\end{aligned}
$$

where the parameters $t_{R}^{\prime}, t_{G}^{\prime}$, and $t_{B}^{\prime}$ belong to the corresponding subsets in (6.7). The first mapping in (6.8) is a ramification, which means that two new parameters $t_{G}^{\prime}$ and $t_{B}^{\prime}$ arise for the tiles $\mathcal{T}_{G}\left(t_{G}^{\prime}\right)$ and $\mathcal{T}_{B}\left(t_{B}^{\prime}\right)$ in the inflated tile $B^{-1} \mathcal{T}_{R}\left(t_{R}\right)$.

Proof. Table 5 shows that $B^{-1} \mathcal{T}_{R}(\mathbf{0})=\mathcal{T}_{R}(\mathbf{0})+\mathcal{T}_{G}\left(\zeta^{2}\right)+\mathcal{T}_{B}\left(\zeta+\zeta^{2}\right)$. If $\mathbf{s}$ is the limit value of a local number $s$, then the shift vector $v(s)=B^{-3} \delta(s)$ transforms to the vector $B^{-1} v(s)=v(\overleftarrow{s})$ under the $B^{-1}$-deflation, and $\zeta \mathbf{s}$ is the limit value of $\overleftarrow{s}$, which proves the lemma.

Proposition 6.2. 1. For every $m \geq 1$, the subset $B^{-m}(0) \subset T[\zeta]$ is the set of parameters $t$ corresponding to all tiles in the tiling $\mathcal{R}^{m-1}$, and

$$
B^{-m}(0)=\{t \in T[\zeta]: \operatorname{deg} t \leq m+1\}
$$

here $\operatorname{deg} t=k$ denotes the degree of $t=t_{1} \zeta+t_{2} \zeta^{2}+\cdots+t_{k} \zeta^{k}$, where $t_{1} t_{2} \ldots t_{k} \in E$ and $t_{k} \neq 0$.

2. For $T[\zeta]$ we have

$$
\begin{aligned}
T[\zeta] & =\left\{t: t_{1} t_{2} \ldots t_{k} \in E, k \in \mathbb{N}\right\} \cap[0|\ldots| 1) \\
& =\left\{t: t_{1} t_{2} \ldots t_{k} \in E, k \in \mathbb{N} \text { and } t_{2}=1 \quad \text { if } t_{1}=1\right\} .
\end{aligned}
$$

In the above notation, the subsets $T_{R}[\zeta), T_{G}[\zeta)$, and $T_{B}[\zeta)$ (see (6.7]) have the form

$$
\begin{aligned}
& T_{R}[\zeta]=\left\{t \in T[\zeta): t_{1}=0, t_{2}=0\right\}, \\
& T_{G}[\zeta]=\left\{t \in T[\zeta): t_{1}=0, t_{2}=1\right\}, \\
& T_{R}[\zeta]=\left\{t \in T[\zeta): t_{1}=1, t_{2}=1\right\} .
\end{aligned}
$$

3. The ramified mapping $B^{-1}: \mathbb{R}^{2} \longrightarrow \mathbb{R}^{2}$ determined by the deflation (6.8) is epimorphic on the set of parameters, $B^{-1} T[\zeta)=T[\zeta)$. 
Proof. Statement 1 follows from the second method of constructing $E$-tilings (see (1.7)) and ramification formula (6.8); statement 2 follows from the inclusion $T[\zeta] \subset[0|\ldots| 1$ ) and Table 5; the last statement follows from the second relation in (6.9) and formulas (6.8).

6.4. Higher order coronas. Table 5 shows the relationship between the types of coronas of the tiling $\mathcal{R}^{\infty}$ and the eleven half-intervals in the tiling

$$
[0|\ldots| 1)^{1}=\left[0, \zeta^{5}\right)_{R}+\cdots+\left[\zeta^{2}, \zeta^{2}+\zeta^{4}\right)_{G}+\cdots+\left[\zeta+\zeta^{2}, 1\right)_{B} .
$$

The half-intervals in the coarser tiling

$$
[0|\ldots| 1)^{0}=\left[0, \zeta^{2}\right)_{R}+\left[\zeta^{2}, \zeta\right)_{G}+\left[\zeta+\zeta^{2}, 1\right)_{B}
$$

correspond to the three types of tiles, $R, G$, and $B$, or to the coronas of zero order. Passing to the coronas of an arbitrary order $r=0,1,2, \ldots$ that are obtained by uniting all local environments of the orders from 0 to $r$, we see that the indicated relationship between them and the tilings of the set $[0|\ldots| 1)$ is preserved.

Proposition 6.3. For every $r$, there exists a finite tiling $[0|\ldots| 1)^{r}$ of the set $[0|\ldots| 1)$ into half-intervals such that arbitrary tiles with parameters $t_{1}$ and $t_{2}$ generate the same coronas of order $r$ if and only if $t_{1}$ and $t_{2}$ belong to the same half-interval of the tiling $[0|\ldots| 1)^{r}$.

Proof. We present two methods of constructing a tiling of $[0|\ldots| 1)$ into half-intervals of $r$-corona types.

Method 1. We start with $t=0$ and successively extend the initial tile $\mathcal{T}_{R}(\mathbf{0})$ by the tiles of the 1st, 2 nd, $\ldots, r$ th environment and calculate their parameters. We denote by $T\left(\mathcal{T}_{R}(\mathbf{0}), r\right) \subset T[\zeta]$ the set of parameters obtained. From $T\left(\mathcal{T}_{R}(\mathbf{0}), r\right)$, we choose parameters $t_{\zeta^{2}}, t_{\zeta}$, and $t_{1}$ closest, respectively, to the endpoints $\zeta^{2}, \zeta$, and 1 from the left but not coinciding with them. Let $\Delta>0$ be the smallest of the corresponding distances. Then $[0, \Delta)$ is a half-interval for all tiles generating $r$-clusters of the same type as the tile $\mathcal{T}_{R}(\mathbf{0})$. Since $\Delta \in T[\zeta]$, there is a tile $\mathcal{T}_{R}(\boldsymbol{\Delta})$ corresponding to it; we apply the same construction to this tile, etc. The number of types of 1-coronas is finite. Consequently, for each $r$, there exist finitely many types of $r$-coronas, and the process terminates after finitely many steps.

Method 2 is based on the dual approach, where the tiling $[0|\ldots| 1)^{r+1}$ is obtained by a refinement of the preceding tiling $[0|\ldots| 1)^{r}$. In particular, the tiling (6.11) is obtained from (6.12) in this way. For this, we must have the tiling (6.12) for $r=0$ and all local numbers from Table 5 with their limit values from Table 4.

At the intersection of the $X$-row and the $Y$-column in Table 6 we present the admissible local numbers $s_{X Y}$ from the $X$-tile to the $Y$-tile. Suppose that the tiling $[0|\ldots| 1)^{r}$ is already constructed. In an arbitrary order, we look through all local numbers in Table 6. If, for example, the local number $s_{R R}$ is chosen, then the point $x$ runs through the interval $\left[0, \zeta^{2}\right)_{R}$ in the tiling $[0|\ldots| 1)^{0}$; see (6.12). We add the division point $x$ to the tiling $[0|\ldots| 1)^{r}$ if its image $x^{\prime}=x+\mathbf{s}_{R R}$ intersects a boundary of a half-interval in $[0|\ldots| 1)^{r} \cap\left[0, \zeta^{2}\right)_{R}$. If the local number has the form $s_{R G}$, then we add the point $x$ if it intersects the image $x^{\prime}=x+\mathbf{s}_{R G}$ of the boundaries of half-intervals in $[0|\ldots| 1)^{r} \cap\left[\zeta^{2}, \zeta\right)_{G}$. In the case of a general $s_{X Y}$, the point $x$ runs through the half-interval $[*, *)_{X}$, and we trace its image $x^{\prime}=x+\mathbf{s}_{X Y}$ as it runs through the half-interval $[*, *)_{Y}$ in the tiling (6.12). Looking through all local numbers occurring in Table 6, we obtain the tiling $[0|\ldots| 1)^{r+1}$. 
TABLE 6

\begin{tabular}{|l|l|l|l|}
\hline & $R$ & $G$ & $B$ \\
\hline$R$ & $\pm 2, \pm 4, \pm 6$, & $2,4,6,7,9,11$ & 17,20 \\
$G$ & $-2,-4,-6,-7,-9,-11$ & \pm 4 & 11,13 \\
$B$ & $-17,-20$ & $-11,-13$ & \\
\hline
\end{tabular}

Proposition 6.4. No two tiles in the tiling $\mathcal{R}^{\infty}$ generate the same r-coronas for all orders $r$.

Proof. This can be proved in the same way as Proposition 6.3. Another, nonconstructive approach, employs relation (0.16), from which it follows that the Rauzy tiling $\mathcal{R}^{\infty}$ is quasiperiodic, and therefore, has no nontrivial translations.

\section{$\S 7$. A parametrization of the plane}

7.1. Distinguished sequences. By definition, we have $\mathcal{R}^{m}=B^{-m} d^{m}$, and the tiling $d^{m}$ is parametrized by sequences in $E^{\infty}$,

$$
\varepsilon=\underbrace{\varepsilon_{0} \ldots \varepsilon_{m-1}}_{m} \quad \varepsilon_{m} \varepsilon_{m+1} \ldots,
$$

where the elements $\varepsilon_{m} \varepsilon_{m+1}=0,10$, or 110 indicate the form of the tile $d_{R}^{m}(*), d_{G}^{m}(*)$, or $d_{B}^{m}(*)$, respectively. Passing from $d^{m}$ to normalized tilings, we obtain the relation

$$
B^{-m} \delta(\varepsilon)=B^{-m} \delta\left(\varepsilon_{0} \ldots \varepsilon_{m-1}\right)+\delta\left(\varepsilon_{m} \varepsilon_{m+1} \ldots\right),
$$

where $\delta\left(\varepsilon_{m} \varepsilon_{m+1} \ldots\right)$ is a point in some tile of the tiling $d^{0}=d_{R}^{0}(0)+d_{G}^{0}(10)+d_{B}^{0}(110)$, because $\mathcal{R}^{0}=d^{0}$ for the zero level, and $B^{-m} \delta\left(\varepsilon_{0} \ldots \varepsilon_{m-1}\right)$ is a shift vector for the tile containing the point $\delta\left(\varepsilon_{m} \varepsilon_{m+1} \ldots\right)$. The separation into two parts in (7.2) makes it necessary to introduce the set of distinguished sequences $\mid E^{\infty}$ of the form

$$
\mid \theta= \begin{cases}\mid \theta_{0} \theta_{1} \ldots & \text { for } m=0, \\ \tau_{1} \ldots \tau_{m} \mid \theta_{0} \theta_{1} \ldots & \text { for } m \geq 1, \quad \tau_{1}=1,\end{cases}
$$

where $\theta=\theta_{0} \theta_{1} \ldots$ and $\tau_{1} \ldots \tau_{m} \theta_{0} \theta_{1} \ldots$ with $\tau_{1}=1$ are sequences in $E^{\infty}$. A distinguished sequence $\mid \theta$ consists of the of $\varepsilon$ - and $\tau$-parts,

$$
\varepsilon(\mid \theta)=\theta_{0} \theta_{1}, \ldots, \quad \tau(\mid \theta)= \begin{cases}0 & \text { for } m=0, \\ \tau_{1} \ldots \tau_{m} & \text { for } m \geq 1,\end{cases}
$$

and has the $t$-parameter

$$
t(\mid \theta)=\left\{\begin{array}{lll}
0 & \text { if } m=0, \quad \theta_{0}=0, \\
\theta_{1} \zeta+\theta_{0} \zeta^{2} & \text { if } m=0, \quad \theta_{0}=1, \\
\tau_{m} \zeta^{3}+\cdots+\tau_{1} \zeta^{m+2} & \text { if } m \geq 1, \quad \theta_{0}=0, \\
\theta_{1} \zeta+\theta_{0} \zeta^{2}+\tau_{m} \zeta^{3}+\cdots+\tau_{1} \zeta^{m+2} & \text { if } m \geq 1, \quad \theta_{0}=1 .
\end{array}\right.
$$

By (7.2), to every distinguished sequence $\mid \theta$, we assign a point of the plane $\mathbb{R}^{\infty}$ by the following rule:

$$
|e:| \theta \longmapsto \Delta(\mid \theta)+\delta(\mid \theta),
$$

where $\Delta(\mid \theta)=B^{-m} \delta(\tau(\mid \theta))$ is a shift vector of a tile for $\mid \theta$ and $\delta(\mid \theta)=\delta(\varepsilon(\mid \theta))$ is a point of a tile $d_{R}^{0}(0), d_{G}^{0}(1)=d_{G}^{0}(10), d_{B}^{0}(3)=d_{B}^{0}(110)$. The degree of the sequence $\mid \theta$ is defined by the relation $m(\mid \theta)=m$. 
7.2. The sets $\mathbb{N} \otimes E^{\infty}$ and $T[\zeta] \otimes E^{\infty}$. The set of distinguished sequences $\mid E^{\infty}$ can be identified with the set $\mathbb{N} \otimes E^{\infty}$ of pairs $(m, \theta)$, where $m \in \mathbb{N}, \theta \in E^{\infty}$, and $\theta=1 \ldots$ if $m \geq 1$. We represent the sequences $\theta$ from $(m, \theta)$ in the form (7.3):

$$
\theta= \begin{cases}\theta_{0} \theta_{1} \ldots & \text { for } m=0 \\ \tau_{1} \ldots \tau_{m} \theta_{0} \theta_{1} \ldots & \text { for } m \geq 1\end{cases}
$$

Then we have the bijection

$$
\mathbb{N} \otimes E^{\infty} \stackrel{\sigma_{1}}{\rightleftharpoons}\left|E^{\infty}:(m, \theta) \mapsto\right| \theta
$$

We consider the diagram

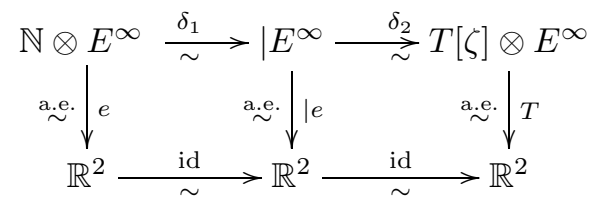

where

$$
\begin{aligned}
& e:(m, \theta) \longmapsto B^{-m} \delta(\theta)=\Delta(m, \theta)+\delta(m, \theta), \\
& \Delta(m, \theta)=B^{-m} \delta\left(\tau_{1} \ldots \tau_{m}\right), \quad \delta(m, \theta)=\delta\left(\theta_{0} \theta_{1} \ldots\right),
\end{aligned}
$$

and we have put $\Delta(0, \theta)=0$;

$$
T[\zeta] \otimes E^{\infty}=\left(T_{R}[\zeta] \times E_{R}^{\infty}\right) \cup\left(T_{G}[\zeta] \times E_{G}^{\infty}\right) \cup\left(T_{B}[\zeta] \times E_{B}^{\infty}\right),
$$

where the sets $T_{X}[\zeta]$ are defined as in (6.10) and the sets $E_{R}^{\infty}, E_{G}^{\infty}$, and $E_{B}^{\infty}$ consist of sequences of the form $0 \ldots, 10 \ldots, 110 \cdots \in E^{\infty}$; the mapping

$$
\sigma_{2}: \mid \theta \longmapsto(t(\mid \theta), \varepsilon(\mid \theta))
$$

is a bijection, and

$$
T:(t, \varepsilon) \longmapsto \Delta(t)+\delta(\varepsilon),
$$

where $\Delta(t)=B^{-m} \delta\left(\tau_{1} \ldots \tau_{m}\right)$ if

$$
t= \begin{cases}\theta_{1} \zeta+\theta_{0} \zeta^{2} & \text { for } m=0, \\ \theta_{1} \zeta+\theta_{0} \zeta^{2}+\tau_{m} \zeta^{3}+\tau_{1} \zeta^{m+2} & \text { for } m \geq 1, \quad \tau_{1}=1 .\end{cases}
$$

We carry the actions of the affine rotations $B^{ \pm}: \mathbb{R}^{2} \longrightarrow \mathbb{R}^{2}$ over to the sets $\mathbb{N} \otimes E^{\infty}$ and $\mid E^{\infty}$ from the first row of the diagram (7.9) as follows:

$$
\begin{aligned}
B^{-1}(m, \theta) & = \begin{cases}\left(0, \theta_{1} \ldots\right) & \text { for } m=0, \quad \theta_{0}=0, \\
1, \theta) & \text { for } m=0, \quad \theta_{0}=1, \\
(m+1, \theta) & \text { for } m \geq 1,\end{cases} \\
B(m, \theta) & = \begin{cases}\left(0,0 \theta_{0} \theta_{1} \ldots\right) & \text { for } m=0, \\
(m-1, \theta) & \text { for } m \geq 1,\end{cases} \\
B^{-1}(\mid \theta) & = \begin{cases}\theta_{0} \mid \theta_{1} \ldots & \text { for } m=0, \\
\tau_{1} \ldots \tau_{m} \theta_{0} \mid \theta_{1} \ldots & \text { for } m \geq 1\end{cases} \\
B(\mid \theta) & = \begin{cases}\mid 0 \theta_{0} \theta_{1} \ldots & \text { for } m=0, \\
\tau_{1} \ldots \mid \tau_{m} \theta_{0} \theta_{1} \ldots & \text { for } m \geq 1 .\end{cases}
\end{aligned}
$$


The actions of $B^{-1}(t, \varepsilon)=\left(B^{-1}(t), B^{-1}(\varepsilon)\right)$ and $B(t, \varepsilon)=(B(t), B(\varepsilon))$ on the set $T[\zeta] \otimes$ $E^{\infty}$ are well defined because diagram (7.9) is commutative. They can be represented in the following explicit form:

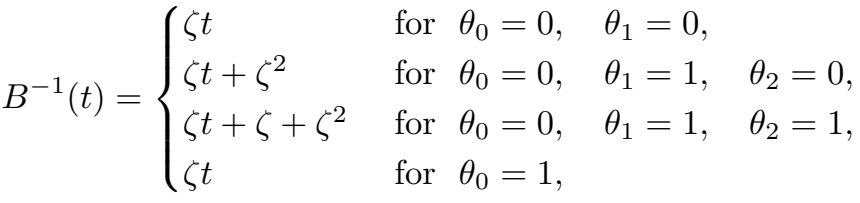

$$
\begin{aligned}
& B^{-1}(\varepsilon)=\theta_{1} \theta_{2} \ldots, \\
& B(t)=\left\{\begin{array}{lll}
0 & \text { for } m=0, \\
\zeta^{-1} t & \text { for } m \geq 1, \quad \theta_{0}=0, & \\
\zeta^{-1} t & \text { for } m \geq 1, \quad \theta_{0}=1, \quad \theta_{1}=0, \quad \tau_{m}=1, \\
\zeta^{-1}\left(t-\zeta^{2}\right) & \text { for } m \geq 1, \quad \theta_{0}=1, \quad \theta_{1}=1,
\end{array}\right. \\
& B(\varepsilon)= \begin{cases}0 \theta_{0} \theta_{1} \ldots & \text { for } m=0, \\
\tau_{m} \theta_{0} \theta_{1} \ldots & \text { for } m \geq 1 .\end{cases}
\end{aligned}
$$

Theorem 7.1. 1. Diagram (7.9) is commutative.

2. Almost everywhere in Lebesgue measure, the mappings e, $\mid e, T$ are bijections onto the set $\mathbb{R}^{2}$.

3. The mappings e, $\mid e, T$ and the corresponding mappings $B^{ \pm 1}$ (see (7.12) and (7.13)) obey the conjugation formulas

$$
B^{ \pm 1} e=e B^{ \pm 1}, \quad B^{ \pm 1}|e=| e B^{ \pm 1}, \quad B^{ \pm 1} T=T B^{ \pm 1} .
$$

4. Let $(t, \varepsilon) \in T[\zeta] \otimes E^{\infty}$, and let the sequence $\varepsilon$ be of the form $\varepsilon_{R}=0, \varepsilon_{G}=10$, or $\varepsilon_{B}=110$. Then the point $T(t, \varepsilon)$ (see (1.7)) of the plane $\mathbb{R}^{2}$ coincides with the center $C(t)$ of the tile $\mathcal{T}_{R}(t), \mathcal{T}_{G}(t)$, or $\mathcal{T}_{R}(t)$, respectively, constructed by the local rules with the help of the algorithm of Subsection 6.2.

Proof. Statements 1 and 3 follow directly from the definitions of the mappings $e, \mid e, T$, and $B^{ \pm 1}$.

We prove 4 . The identity

$$
T(t, \varepsilon)=C(t)
$$

for $t=0$ follows from the definition (7.10) of the mapping $T$ and the algorithm (see Subsection 6.2) of the layerwise construction of the tiling $\mathcal{R}^{\infty}$. For arbitrary values of $t$, formula (7.15) is proved by induction on $m$ : we apply the mapping $B^{-1}$ (see (7.13)) to the left-hand side of (7.15) and the affine rotation $B^{-1}$ as in (2.4) to the right-hand side. The transition $m \mapsto m+1$ is obtained with the help of Proposition 6.1 and the formula for $B^{-m}(0)$ in Proposition 6.2.

We begin the proof of Statement 2 with the tilings $d^{m}=\delta\left(E^{m}\right)$ of the torus $\mathbb{T}^{2}$ (see (1.5) and (4.4)). If a point $x$ is such that $x \in \mathbb{T}^{2}$ and $x \notin \partial d^{m}=\bar{d}^{m} \backslash d^{m}$ (the boundary of $d^{m}$ ), then $x$ belongs to only one tile in $d^{m}$, and consequently, its index $\bar{i}, \bar{j}, \bar{k}$ in (1.5) is determined uniquely. The diameters of the tiles of $d^{m}$ tend to zero as $m \rightarrow \infty$. Therefore, if $x$ does not belong to the set $\partial^{\infty}=\bigcup_{m=0}^{\infty} \partial d^{m}$, then there is a unique infinite sequence $\varepsilon \in E^{\infty}$ assigned to such $x, x \mapsto \varepsilon$. Since the Lebesgue measure of the set $\partial^{\infty}$ is zero, we have a mapping $E^{\infty} \rightarrow d^{m}$; this mapping is invertible almost everywhere, and consequently, is a bijection.

We prove that the mapping $e: \mathbb{N} \otimes E^{\infty} \longrightarrow \mathbb{R}^{2}$ is also a bijection almost everywhere. For this, we apply the approximation $\mathcal{R}^{0} \subset \mathcal{R}^{1} \subset \cdots \subset \mathcal{R}^{m} \subset \cdots$ of the Rauzy tiling 
$\mathcal{R}^{\infty}$ of $\mathbb{R}^{2}$ by the finite tilings $\mathcal{R}^{m}=B^{-m} d^{m}$. Since the set $\mathbb{N} \otimes E^{\infty}$ admits the tiling

$$
\mathbb{N} \otimes E^{\infty}=\left(0 \times E^{\infty}\right) \cup\left(1 \times E_{1}^{\infty}\right) \cup \cdots \cup\left(m \times E_{1}^{\infty}\right) \cup \ldots,
$$

where $E_{1}^{\infty}$ denotes the subset of sequences of the form $\varepsilon=1 \cdots \in E^{\infty}$, the mappings $E_{1}^{\infty} \longrightarrow d^{m} \backslash B d^{m-1}$ are bijections almost everywhere by (7.9), and $B^{-m}$ : $d^{m} \backslash B d^{m-1} \longrightarrow \mathcal{R}^{m} \backslash \mathcal{R}^{m-1}$ is an isomorphism, we see that the mapping $e$ splits into layers as follows:

$$
\begin{array}{lll}
e: 0 \times E^{\infty} & \stackrel{\text { a.e. }}{\rightleftharpoons} & \mathcal{R}^{0}, \\
e: 1 \times E_{1}^{\infty} & \stackrel{\text { a.e. }}{\rightleftharpoons} & \mathcal{R}^{1} \backslash \mathcal{R}^{0}, \\
& \ldots & \\
e: 1 \times E_{1}^{\infty} & \stackrel{\text { a.e. }}{\rightleftharpoons} & \mathcal{R}^{m} \backslash \mathcal{R}^{m-1}, \\
& \ldots &
\end{array}
$$

Since, as a set, the tiling $\mathcal{R}^{\infty}$ coincides with $\mathbb{R}^{2} \backslash \partial \mathcal{R}^{\infty}$ and the boundary $\partial \mathcal{R}^{\infty}$ has zero measure, (7.16) shows that the mapping $e$ is a bijection almost everywhere. Similar statements concerning the mappings $\mid e, T$ follow from the fact that diagram (7.9) is commutative.

The commutative diagram

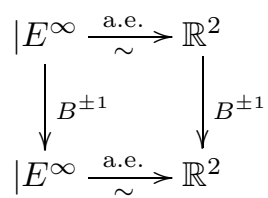

shows that, almost everywhere, to each point $x \in \mathbb{R}^{2}$ the Rauzy tiling $\mathcal{R}^{\infty}$ assigns the code of $x$,

$$
r=\left|e^{-1}: \quad \mathbb{R}^{2} \stackrel{\text { a.e. }}{\rightleftharpoons}\right| E^{\infty}: x \longmapsto|\varepsilon=| \varepsilon(x),
$$

where $\left|\varepsilon=\ldots \varepsilon_{-2} \varepsilon_{-1}\right| \varepsilon_{0} \varepsilon_{1} \ldots$ is a distinguished sequence in $\mid E^{\infty}$; this can be done by the following rule. We consider the following two sequences.

1) The infinite sequence

$$
x_{0}=B^{-0} x, \quad x_{1}=B^{-1} x, \ldots, x_{n}=B^{-n} x, \ldots,
$$

which determines $\varepsilon_{n}=0$ if $x_{n} \in \mathcal{R}_{R}(t)$ and $\varepsilon_{n}=1$ otherwise, where $\mathcal{R}_{R}(t)$ are tiles in the Rauzy tiling

$$
\mathcal{R}^{\infty}=\sum_{t \in T[\zeta]} \mathcal{R}_{X(t)}(t),
$$

and $X(t)=R, G, B$ is the form of tiles determined by the parameter $t$.

2) The finite sequence

$$
x_{-1}=B^{1} x, \quad x_{-2}=B^{2} x, \ldots,
$$

which terminates as soon as the point $x_{-n}$ is in the tiling of zero level $\mathcal{R}^{0}=\mathcal{R}_{R}^{0}(0) \cup$ $\mathcal{R}_{G}^{0}\left(\zeta^{2}\right) \cup \mathcal{R}_{B}^{0}\left(\zeta+\zeta^{2}\right)$. The elements $\varepsilon_{-n}=0$ or 1 are defined similarly. The matrix $B$ has the eigenvalues $|\alpha|=\left|\alpha^{\prime}\right|<1$, and the closure $\overline{\mathcal{R}}^{0}$ contains a neighborhood of zero. Therefore, the process (7.20) terminates after finitely many steps depending on $x$.

The codes $r(x)=\left|\varepsilon(x)=\ldots \varepsilon_{-2} \varepsilon_{-1}\right| \varepsilon_{0} \varepsilon_{1} \ldots$ are compatible,

$$
r\left(B^{ \pm 1} x\right)=B^{ \pm 1} r(x),
$$

with the actions of the affine rotations $B^{ \pm 1}$ (see (2.4)) on the points $x$ and the shifts $B^{ \pm 1}$ (see (7.12)) of their codes $r(x)$. 


\section{REFERENCES}

[1] V. G. Zhuravlev, Rauzy tilings and bounded remainder sets on a torus, Zap. Nauchn. Sem. S.-Peterburg. Otdel. Mat. Inst. Steklov. (POMI) 322 (2005), 83-106; English transl., J. Math. Sci. (N.Y.) 137 (2006), no. 2, 4658-4672. MR.2138453 (2006b:11094)

[2] _ One-dimensional Fibonacci tilings, Izv. Ross. Akad. Nauk Ser. Mat. 71 (2007), no. 2, 89-122; English transl., Izv. Math. 71 (2007), no. 2, 307-340. MR2316983 (2008i:11042)

[3] _ On additivity property of the complexity function related to Rauzy tiling, Analytic and Probabilistic Methods in Number Theory, TEV, Vilnius, 2007, pp. 240-254. MR2397156 (2009g:52040)

[4] V. G. Zhuravlev and A. V. Maleev, The complexity function and forsing in two-dimensional quasiperiodic Rauzy tiling, Kristallografiya 52 (2007), no. 4, 610-616. (Russian)

[5] _ The layer-by-layer growth of a quasiperiodic Rauzy tiling, Kristallografiya 52 (2007), no. 2, 204-210. (Russian)

[6] _ Quasiperiods of layer-by-layer growth of the Rauzy tiling, Kristallografiya 52 (2007), no. 1, 7-14. (Russian)

[7] (2008), no. 6, 978-986. (Russian)

[8] Construction of the two-dimensional quasiperiodic Rauzy tiling by means of similarity transformation, Kristallografiya 54 (2009), no. 3, 389-399. (Russian)

[9] A. V. Maleev, A. V. Shutov, and V. G. Zhuravlev, Quasiperiodic tilings of plane constructed on the basis of cubic irrationalities, 27th N. V. Belov Scientific Readings, Nizhni Novgorod, 2008, pp. 2931. (Russian)

[10] S. Akiyama, Self affine tiling and Pisot numeration system, Number Theory and its Applications (Kyoto 1997), Dev. Math., vol. 2, Kluwer, Dordrecht, 1999, pp. 7-17. MR.1738803 (2001b:11094)

[11] _ Cubic Pisot units with finite beta expansions, Algebraic Number Theory and Diophantine Analysis (Graz, 1988), de Gruyter, Berlin, 2000, pp. 11-26. MR.1770451(2001i:11095)

[12] P. Arnoux, V. Berthé, and S. Ito, Discrete plane, $\mathbb{Z}^{2}$-actions, Jacobi-Perron algorithm and substitutions, Ann. Inst. Fourier (Grenoble) 52 (2002), 305-349. MR.1906478 (2004d:37012)

[13] N. P. Fogg, Substitutions in dynamics, arithmetics and combinatorics, Lecture Notes in Math., vol. 1794, Springer-Verlag, Berlin, 2002. MR1970385 (2004c:37005)

[14] S. Ito and M. Ohtsuki, Modified Jacobi-Perron algorithm and generating Markov partitions for special hyperbolic toral automorphisms, Tokyo J. Math. 16 (1993), no. 2, 441-472. MR1247666 (95g:58167)

[15] S. Ito, J. Fujii, H. Higashino, and S. Yasutomi, On simultaneous approximation to $(\alpha, \alpha)^{2}$ with $\alpha^{3}+k \alpha-1=0$, J. Number Theory 99 (2003), 255-283. MR.1968452 (2004a:11063)

[16] Y. Meyer, Algebraic numbers and harmonic analysis, North-Holland Math. Library, vol. 2, NorthHolland, Amsterdam-London, 1972. MR0485769 (58:5579)

[17] R. V. Moody, Meyer sets and their duals, The Mathematics of Long-Range Aperiodic Order (Waterloo, ON, 1995), NATO Adv. Sci. Inst. Ser. C Math. Phys., vol. 489, Kluwer, Dordrecht, 1997, pp. 403-441. MR.1460032 (98e:52029)

[18] G. Rauzy, Nombres algébriques et substitutions, Bull. Soc. Math. France 110 (1982), 147-178. MR0667748(84h:10074)

Vladimir State Pedagogical University, Stroitelei 11, Vladimir 600024, Russia

E-mail address: vzhuravlev@mail.ru

Received 1/APR/2009

Translated by B. BEKKER 